\title{
Rotational Light Curves of Jupiter from Ultraviolet to Mid-infrared and Implications for Brown Dwarfs and Exoplanets
}

\author{
Huazhi Ge (葛華志) ${ }^{1}$ (1D, Xi Zhang ${ }^{1}$, Leigh N. Fletcher ${ }^{2}$ (i), Glenn S. Orton ${ }^{3}$, James Sinclair ${ }^{3}$, Josh Fernandes ${ }^{3}$, Tom Momary ${ }^{3}$, \\ Yasumasa Kasaba ${ }^{4}$, Takao M. Sato ${ }^{5}$, and Takuya Fujiyoshi ${ }^{6}$ \\ ${ }^{1}$ Department of Earth and Planetary Sciences, University of California Santa Cruz, Santa Cruz, CA 95064, USA; huazhige@ucsc.edu \\ ${ }^{2}$ Department of Physics \& Astronomy, University of Leicester, University Road, Leicester LE1 7RH, UK \\ ${ }^{3}$ Jet Propulsion Laboratory, California Institute of Technology, 4800 Oak Grove Drive, Pasadena, CA 91109, USA \\ ${ }^{4}$ Planetary Plasma and Atmospheric Research Center, Tohoku University, Aramaki-aza-Aoba 6-3, Aoba, Sendai, Miyagi 980-8578, Japan \\ ${ }^{5}$ Institute of Space and Astronomical Science, Japan Aerospace Exploration Agency, 3-1-1, Yoshinodai, Chuo-ko, Sagamihara, Kanagawa 252-5210, Japan \\ ${ }^{6}$ Subaru Telescope, National Astronomical Observatory of Japan, National Institutes of Natural Sciences, 650 North A'ohoku Place, Hilo, HI 96720, USA \\ Received 2018 September 6; revised 2018 December 16; accepted 2019 January 1; published 2019 February 1
}

\begin{abstract}
Rotational modulations are observed on brown dwarfs and directly imaged exoplanets, but the underlying mechanism is not well understood. Here we analyze Jupiter's rotational light curves at 12 wavelengths from the ultraviolet (UV) to the mid-infrared (mid-IR). The peak-to-peak amplitudes of Jupiter's light curves range from subpercent to $4 \%$ at most wavelengths, but the amplitude exceeds $20 \%$ at $5 \mu \mathrm{m}$, a wavelength sensing Jupiter's deep troposphere. Jupiter's rotational modulations are primarily caused by discrete patterns in the cloudless belts instead of the cloudy zones. The light-curve amplitude is controlled by the sizes and brightness contrasts of the Great Red Spot (GRS), expansions of the North Equatorial Belt (NEB), patchy clouds in the North Temperate Belt (NTB), and a train of hot spots in the NEB. In reflection, the contrast is controlled by upper tropospheric and stratospheric hazes, clouds, and chromophores in the clouds. In thermal emission, the small rotational variability is caused by the spatial distribution of temperature and opacities of gas and aerosols; the large variation is caused by the $\mathrm{NH}_{3}$ cloud holes and thin-thick clouds. The methane-band light curves exhibit opposite-shape behavior compared with the UV and visible wavelengths, caused by a wavelength-dependent brightness change of the GRS. Light-curve evolution is induced by periodic events in the belts and longitudinal drifting of the GRS and patchy clouds in the NTB. This study suggests several interesting mechanisms related to distributions of temperature, gas, hazes, and clouds for understanding the observed rotational modulations on brown dwarfs and exoplanets.
\end{abstract}

Key words: brown dwarfs - methods: data analysis - planets and satellites: individual (Jupiter) - techniques: photometric

\section{Introduction}

Rotational photometric variabilities have been observed on brown dwarfs (e.g., Radigan et al. 2012; Yang et al. 2016; Apai et al. 2017), exoplanets (Zhou et al. 2016), and solar system gas giants (e.g., Gelino \& Marley 2000; Karalidi et al. 2015; Simon et al. 2016). The observed rotational variabilities range from subpercent to tens of percent (e.g., Artigau et al. 2009; Radigan et al. 2012; Heinze et al. 2013; Metchev et al. 2015). The observed light curves also show short-term and long-term evolution and wavelength-dependent signatures (e.g., Buenzli et al. 2012; Radigan et al. 2012; Yang et al. 2016; Apai et al. 2017). These observations indicate that these substellar atmospheres possess complex meteorology that evolves with time.

The current leading interpretation is that patchy clouds at different pressure levels cause the observed photometric variability (e.g., Radigan et al. 2012; Apai et al. 2013, 2017). Observations and theoretical models suggest that various types of clouds, such as silicates, salts, and metals, can form on brown dwarfs and close-in exoplanets (e.g., Ackerman \& Marley 2001; Burgasser et al. 2002; Morley et al. 2012; Tan \& Showman 2017; Powell et al. 2018). Recent studies suggest the existence of water clouds on one of the nearest type-Y brown dwarfs (WISE 0855), which has an effective temperature similar to Jupiter's water cloud level (Skemer et al. 2016; Morley et al. 2018). Previous studies have investigated the relationship between patchy cloud patterns and the photometric variability. The thin-thick cloud scenario proposed in Apai et al. (2013) could explain the pressure-dependent behaviors of multiwavelength light curves. Apai et al. (2017) suggested that long-term light-curve evolution on brown dwarfs is generated by beating patterns between planetary waves influencing the distribution of clouds. Morley et al. (2014) suggested that patchy clouds could generate large variability in the nearinfrared (near-IR) on objects warmer than $375 \mathrm{~K}$. For objects cooler than $375 \mathrm{~K}$, a larger-amplitude variability could be produced in the mid-IR due to water condensation. Alternatively, it was suggested that temperature fluctuations could also generate rotational photometric variabilities (e.g., Morley et al. 2014; Robinson \& Marley 2014; Zhang \& Showman 2014). Unfortunately, due to the difficulty of spatially resolving the atmospheres of remote brown dwarfs and exoplanets, the mechanisms behind the rotational photometric variability are still under debate.

With spatially resolved maps, gas-giant atmospheres in the solar system shed light on the mechanisms behind rotational light curves. Previous studies indicated that Jupiter and Neptune exhibit rotational modulations (Gelino \& Marley 2000; Karalidi et al. 2015; Simon et al. 2016; Stauffer et al. 2016). The magnitudes of the photometric variabilities are also wavelength-dependent, ranging from the subpercent level to $\sim 10 \%$ at different wavelengths on Jupiter and Neptune. The atmosphere patterns can be retrieved from light-curve evolution. For instance, Karalidi et al. (2015) provided a mapping tool to retrieve the Great Red Spot (GRS) and $5 \mu \mathrm{m}$ hot spots 
on Jupiter from the light curves of two consecutive rotations at the ultraviolet (UV) and near-IR. Simon et al. (2016) retrieved the jet speeds on Neptune using the power spectra of 50 day light curves at the visible wavelengths from Kepler observation.

However, most previous studies on rotational modulations of Jupiter and Neptune focused on the wavelengths dominated by sunlight reflection (Gelino \& Marley 2000; Karalidi et al. 2015; Simon et al. 2016; Stauffer et al. 2016). The thermal-emission light curves have not been thoroughly investigated yet. Because the light curves of free-floating brown dwarfs are observed in thermal emission, the IR light curves of Jupiter might provide a better insight to understand the observed rotational variability of exoplanets and brown dwarfs. Furthermore, weather patterns on Jupiter might be similar to the cold Y-type brown dwarfs (effective temperature 300-500 K; e.g., Skemer et al. 2016; Morley et al. 2018), which might exhibit zonally banded patterns and storms (Zhang \& Showman 2014).

Jupiter's thermal-emission observations have provided high spatial resolution maps of temperature and chemical compositions $\left(\mathrm{NH}_{3}, \mathrm{PH}_{3}\right.$, hydrocarbons, and aerosols) at multiple pressure levels from $\mathrm{NH}_{3}$ cloud condensation level to the stratosphere (e.g., Orton et al. 1991; Fletcher et al. 2016; Simon et al. 2016). Following the convention, in this paper, we use "cloud" to represent the condensed volatiles such as $\mathrm{NH}_{3}$, $\mathrm{NH}_{4} \mathrm{HS}$, and $\mathrm{H}_{2} \mathrm{O}$. "Chromophore" stands for the color agents (such as the reddish species) in the GRS and the other regions in and above the $\mathrm{NH}_{3}$ clouds (Carlson et al. 2016; Sromovsky et al. 2017). We use "haze" to stand for the particles above and close to the main $\mathrm{NH}_{3}$ cloud top, including upper tropospheric and stratospheric aerosol particles (i.e., West \& Smith 1991; West et al. 2004; Zhang et al. 2013; Fletcher et al. 2016). The term "aerosol" is a general name for all particles including hazes. The distributions of deep $\mathrm{H}_{2} \mathrm{O}$ clouds (8-5 bars), $\mathrm{NH}_{4} \mathrm{HS}$ (2-1.5 bars), and $\mathrm{NH}_{3}$ clouds (0.7-0.3 bars) in the troposphere (West et al. 2004); hazes in the upper troposphere and stratosphere; and chromophores in and above the $\mathrm{NH}_{3}$ clouds have all been well observed and studied for decades (e.g., Ferris \& Ishikawa 1987; Taylor et al. 2004; West et al. 2004; Carlson et al. 2016; Sromovsky et al. 2017). These data allow us to thoroughly investigate the underlying mechanism of the rotational light curves of Jupiter in thermal emission and quantify the important roles of temperature, gas, and clouds.

In this study, we analyze Jupiter's light curves at both reflection and thermal-emission wavelengths and provide interpretations of what controls the light-curve amplitudes and wavelength-dependent behaviors. We construct the sunlight-reflected light curves from the radiance maps imaged by the Wide Field Camera 3 (WFC3)/UVIS on the Hubble Space Telescope (HST) from the UV to the near-IR wavelengths in nine channels. The thermal-emission light curves are constructed from images obtained by the COMICS instrument on the Subaru Telescope (Kataza et al. 2000), the VISIR instrument on the Very Large Telescope (VLT; Lagage et al. 2004), and the SpeX instrument on NASA's Infrared Telescope Facility (IRTF) in three mid-IR channels. We describe the data acquisition in Section 2 and light-curve construction in Section 3. In Section 3, we also introduce a new method to quantitatively estimate the contribution of the rotational modulation from each latitude to identify the locations of critical discrete patterns. In Section 4, we overview the important discrete patterns and periodic events that are responsible for Jupiter's rotational modulation. Section 5 discusses how the light-curve amplitudes, shapes, and evolution are influenced by the sizes, brightnesses, locations, and evolution of discrete patterns. We will also discuss the underlying physical and chemical mechanisms. Section 6 describes the implications of this study on the multiwavelength rotational variabilities of brown dwarfs and directly imaged exoplanets. We conclude this study in Section 7.

\section{Data}

\subsection{Reflection Data}

We use the global maps of Jupiter in reflected sunlight obtained during the Outer Planets Atmosphere Legacy (OPAL) program by the HST WFC3 (Simon et al. 2015). The OPAL data cover a large wavelength range from the UV to the near-IR in nine channels. The images at each wavelength are reduced via the standard Hubble processing pipeline (Section 2.2 in Simon et al. 2015). Jupiter is mapped from the latitude of $-79.8^{\circ} \mathrm{S}$ to $+79.8^{\circ} \mathrm{N}$ with a resolution of $\sim 10$ pixels per degree (corresponding to $\sim 120 \mathrm{~km}$ at the equator to $\sim 600 \mathrm{~km}$ at the high latitudes). Information on the wavelengths, filters, observational dates, and main opacity sources is listed in Table 1. More detailed information about the data is provided on the official OPAL website (e.g., Simon et al. 2015): https:// archive.stsci.edu/prepds/opal/.

Since 2014, the OPAL program has provided multiwavelength maps of two of Jupiter's rotations each year. But Jupiter's moons (e.g., Io) cast shadows on some maps (e.g., all maps in rotation B of cycle 23). Also, some maps possess residual-fringe features (e.g., $889 \mathrm{~nm}$ map in rotation A of cycle 22). The major satellite shadows and residual fringes generate photometric variabilities that we are not interested in. Thus, we excluded these OPAL maps. We chose rotation B from cycle 22 at 02:00-12:30 UT on 2015 January 19, rotation A from cycle 23 at 09:35-18:04 UT on 2016 February 9, and rotation B from cycle 24 in 2017 April from the OPAL database. The $1 \mathrm{yr}$ interval between these maps allows us to investigate long-term light-curve evolution on Jupiter.

The observations in reflected sunlight at different wavelengths are sensitive to different pressure layers and compositions in Jupiter's atmosphere in and above the $\mathrm{NH}_{3}$ cloud layer. The UV $(0.275 \mu \mathrm{m})$ maps reveal the distributions of upper tropospheric hazes at the equator and midlatitude regions and stratospheric hazes at high latitudes (West et al. 2004; Zhang et al. 2013). At the equator and the midlatitude regions, the UV observation probes the troposphere to the 100 mbar level (Vincent et al. 2000). Due to abundant stratospheric hazes and the limb-darkening effects, the effective pressure layer (where optical depth is $\sim 1$ ) is about tens of mbar in the high-latitude region. The visible-light observations $(0.343,0.395,0.467$, $0.502,0.547,0.631$, and $0.658 \mu \mathrm{m}$ ) are also sensitive to the chromophores in the $\mathrm{NH}_{3}$ clouds, probing pressure levels of a few hundred mbar. In the strong methane band at $0.889 \mu \mathrm{m}$, the observations would probe the 600 mbar pressure level in the absence of clouds (West et al. 2004). However, in the presence of patchy and discrete clouds, the effective pressure levels are much higher in altitude, being strongly affected by the cloud and haze opacities. 
Table 1

Observational Data Information

\begin{tabular}{|c|c|c|c|c|c|c|c|c|}
\hline $\begin{array}{l}\text { Central } \\
\text { Wavelength }\end{array}$ & $\begin{array}{l}\text { Filter } \\
\text { Width }\end{array}$ & $\begin{array}{l}\text { Instrument } \\
\text { /Telescope }\end{array}$ & Date & Minnaert $k$ & $C_{0}$ & $C_{1}$ & Category & Opacity \\
\hline $0.275 \mu \mathrm{m}$ & & WFC3/HST & $\begin{array}{l}2016 \text { Feb } 9 \\
2017 \text { Apr } 3\end{array}$ & $\begin{array}{l}0.520 \\
0.520\end{array}$ & & & UV & Haze absorption \\
\hline $0.343 \mu \mathrm{m}$ & & $\mathrm{WFC} 3 / H S T$ & $\begin{array}{c}2015 \text { Jan } 19 \\
2017 \text { Apr } 3\end{array}$ & $\begin{array}{l}0.850 \\
0.850\end{array}$ & & & Blue & Chromophores \\
\hline $0.395 \mu \mathrm{m}$ & & $\mathrm{WFC} 3 / H S T$ & $\begin{array}{l}2015 \text { Jan } 19 \\
2016 \text { Feb 9, } \\
2017 \text { Apr } 3\end{array}$ & $\begin{array}{l}0.850 \\
0.850 \\
0.850\end{array}$ & & & Blue & Chromophores \\
\hline $0.467 \mu \mathrm{m}$ & & $\mathrm{WFC} 3 / H S T$ & $\begin{array}{l}2016 \text { Feb } 9 \\
2017 \text { Apr } 3\end{array}$ & $\begin{array}{l}0.950 \\
0.950\end{array}$ & & & Blue & Chromophores \\
\hline $0.502 \mu \mathrm{m}$ & & $\mathrm{WFC} 3 / H S T$ & $\begin{array}{l}2015 \text { Jan } 19 \\
2016 \text { Feb } 9 \\
2017 \text { Apr } 3\end{array}$ & $\begin{array}{l}0.950 \\
0.950 \\
0.950\end{array}$ & & & Green & Chromophores \\
\hline $0.547 \mu \mathrm{m}$ & & WFC3/HST & 2016 Feb 9 & 0.970 & & & Green & Chromophores \\
\hline $0.631 \mu \mathrm{m}$ & & $\mathrm{WFC} 3 / H S T$ & $\begin{array}{l}2015 \text { Jan } 19 \\
2016 \text { Feb 9, } \\
2017 \text { Apr } 3\end{array}$ & $\begin{array}{l}0.999 \\
0.999 \\
0.999\end{array}$ & & & Red & Chromophores \\
\hline $0.658 \mu \mathrm{m}$ & & $\mathrm{WFC} 3 / H S T$ & $\begin{array}{l}2015 \text { Jan } 19 \\
2016 \text { Feb 9, } \\
2017 \text { Apr } 3\end{array}$ & $\begin{array}{l}0.999 \\
0.999 \\
0.999\end{array}$ & & & Red & Chromophores \\
\hline $0.889 \mu \mathrm{m}$ & & $\mathrm{WFC} 3 / H S T$ & $\begin{array}{l}2015 \text { Jan } 19 \\
2016 \text { Feb 9, } \\
2017 \text { Apr } 3\end{array}$ & $\begin{array}{l}1.000 \\
1.000 \\
1.000\end{array}$ & & & Near-IR & $\mathrm{CH}_{4}$ \\
\hline $5.1 \mu \mathrm{m}$ & $0.25 \mu \mathrm{m}$ & SpeX/IRTF & 2016 May $11-12$ & & & & Mid-IR & Atmosphere window \\
\hline $8.59 \mu \mathrm{m}$ & $0.42 \mu \mathrm{m}$ & VISIR/VLT & 2016 Feb 15-16 & & 0.032 & 0.120 & Mid-IR & $\mathrm{PH}_{3}$ \\
\hline $8.80 \mu \mathrm{m}$ & $0.8 \mu \mathrm{m}$ & COMICS/Subaru & 2016 Jan 24-25 & & 0.083 & 0.119 & Mid-IR & $\mathrm{PH}_{3}$ \\
\hline $10.50 \mu \mathrm{m}$ & $1.0 \mu \mathrm{m}$ & COMICS/Subaru & 2016 Jan $24-25$ & & 0.152 & 0.202 & Mid-IR & $\mathrm{NH}_{3}$ \\
\hline $10.77 \mu \mathrm{m}$ & $0.19 \mu \mathrm{m}$ & VISIR/VLT & 2016 Feb 15-16 & & 0.102 & 0.160 & Mid-IR & $\mathrm{NH}_{3}$ \\
\hline
\end{tabular}

Note. Limb-darkening coefficient at reflected sunlight and thermal-emission wavelengths, Minnaert $k, C_{0}$, and $C_{1}$ are shown in the table.

\subsection{Thermal-emission Data}

Thermal-emission snapshots of Jupiter's maps were provided by the VISIR instrument (Lagage et al. 2004) on the VLT in 2016 February (program ID 096.C-0091), the COMICS instrument (Kataza et al. 2000) on the Subaru Telescope in 2016 January (program ID S16B-049), and the SpeX instrument on NASA's IRTF in 2016 May (program ID 2016A-022). Both the VLT and Subaru have about $8 \mathrm{~m}$ diameter primary mirrors, providing sufficient spatial resolution to resolve discrete features on Jupiter's disk. Both COMICS and VISIR observe Jupiter in multiple wavelengths from 7 to $25 \mu \mathrm{m}$; we have selected wavelengths near $8.7-8.8 \mu \mathrm{m}$ (sensing $\sim 600 \mathrm{mbar}$ tropospheric $\mathrm{PH}_{3}$, aerosol opacity, and temperature) and 10.3-10.7 $\mu \mathrm{m}$ (sensing tropospheric ammonia and temperature near $400-500$ mbar) for this study. These are supplemented by observations at $5 \mu \mathrm{m}$ sensing Jupiter's mid-tropospheric emission, with condensation clouds in the $1-4$ bar range appearing in silhouette against this bright thermal background. According to some recent studies (e.g., Bjoraker et al. 2015), it was suggested that the observed pressure broadening of lines at $5 \mu \mathrm{m}$ implies the presence of a water cloud, but the narrowband imaging used in this study blends together the contributions from clouds and gases to provide a broad and extended contribution function over the 1-4 bar range. The SpeX/IRTF observations use the smaller $3 \mathrm{~m}$ primary mirror. Figure 1 shows these three different wavelengths; for each wavelength, we have six disk images acquired through two consecutive nights. The spatial resolution varies from 0 !" 2 to 0 "! 4 ( $\sim 700$ to $\sim 1400 \mathrm{~km}$ on Jupiter). Although the thermal-emission maps of Jupiter have coarser resolution than the reflection maps, the spatial resolution is good enough to resolve most small-scale discrete patterns, such as ovals in the southern hemisphere and $5 \mu \mathrm{m}$ hot spots (Figure 1). We use a similar data reduction procedure as described by Fletcher et al. (2009) and Fletcher et al. (2016) for the bad-pixel removal, flat fielding, limb fitting, and cylindrical map projection. Observations at 8.8 and $10.5 \mu \mathrm{m}$ were radiometrically calibrated to match the results from Cassini's Composite Infrared Spectrometer (CIRS) during its Jupiter flyby in 2000 (Fletcher et al. 2016). We note that Jupiter observations with VISIR overfill the $38^{\prime \prime} \times 38^{\prime \prime}$ field of view, meaning that one of the poles is always omitted from the observation in Figure 1, whereas the field of view of COMICS $\left(42^{\prime \prime} \times 32^{\prime \prime}\right)$ is sufficient to capture the whole disk of Jupiter in a two-point mosaic. Taken together, these three wavelengths are sensitive to the distributions of temperature, gases (e.g., $\mathrm{NH}_{3}, \mathrm{PH}_{3}$, and $\mathrm{CH}_{3} \mathrm{D}$ ), hazes, and clouds in the mid- and upper troposphere of Jupiter.

\section{Mosaicked Map and Light-curve Construction}

Following the methods in Gelino \& Marley (2000) and Cowan \& Agol (2008), we construct Jupiter's light curves from Jupiter's cylindrical global maps using carefully characterized wavelength-dependent limb-darkening properties.

\subsection{Reflection Light-curve Construction}

At wavelengths sensing reflected sunlight, the OPAL data set provides the cylindrical global maps, as well as the limbdarkening formula and coefficients. The reflection light curves 


\section{VISIR/VLT 2016-02-15/16}

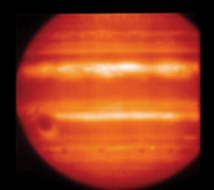

$04: 54: 11$ 15th

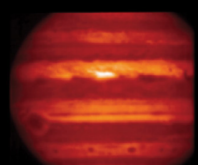

04:48:49 15th

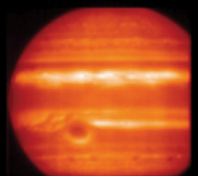

05:41:21 15th

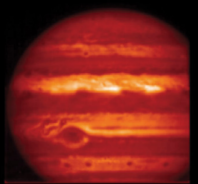

05:35:53 15th

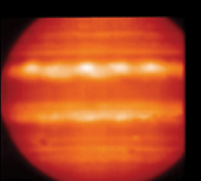

08:19:05 15th

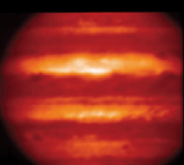

$08: 14: 17$ 15th

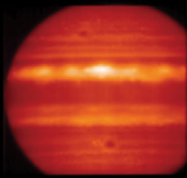

05:21:13 16th

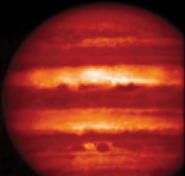

05:15:37 16th

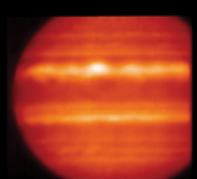

07:37:01 16th

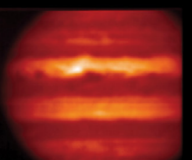

05:15:37 16th

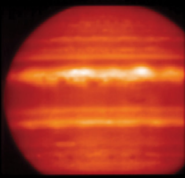

10.77 Micron

$400 \mathrm{mbar}$

$08: 25: 45$ 16th

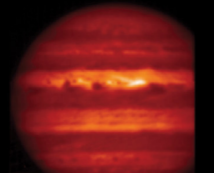

08:21:11 16th

\subsection{Micron}

650 mbar

\section{COMICS/Subaru 2016-01-24/25}

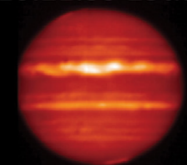

$10: 18: 13$ 25th

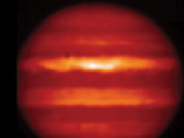

10:21:55 25th

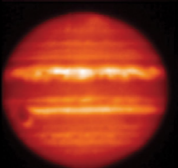

12:32:55 25th

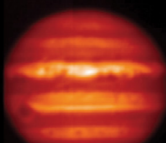

$12: 36: 40$ 25th

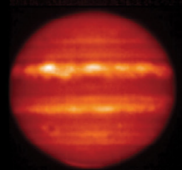

10:41:45 24th

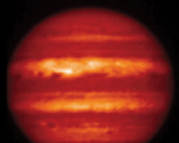

$10: 46: 03$ 24th

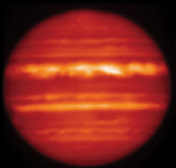

16:05:39 24th

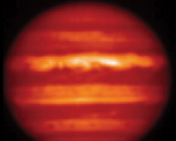

16:02:04 24th

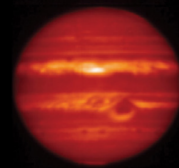

14:29:03 25th

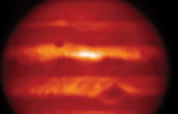

14:32:43 25th

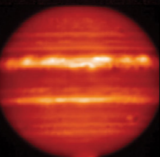

\subsection{Micron}

400 mbar

\section{3:04:16 24th}

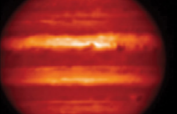

8.80 Micron

$650 \mathrm{mbar}$

13:08:10 24th

\section{SpeX/NASA IRTF 2016-05-11/12}

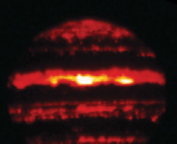

05:18:23 12th

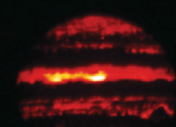

$08: 47: 33$ 11th

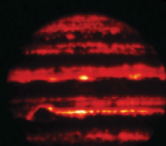

$06: 15: 30$ 11th

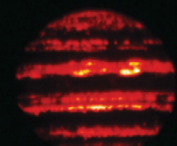

04:56:31 11th

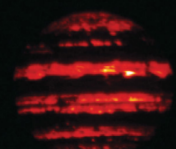

$08: 29: 42$ 12th

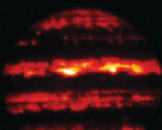

\subsection{Micron} 1000 mbar

5000 mbar

06:36:01 12th

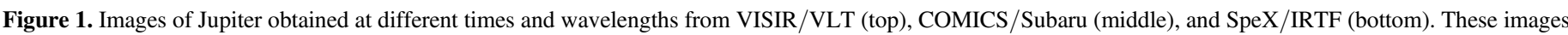
are combined to generate global cylindrical radiance maps in this study after limb-darkening correction.

can be directly constructed via moving a simulated aperture scanning through the global radiance maps. The aperture is $180^{\circ}$ longitude wide and covers all latitudes. As the planet rotates in the aperture, we apply the limb-darkening effect on the radiance map. The light-curve flux is calculated by integrating the pixel radiance weighted by its projected area on a disk within the aperture,

$$
\begin{gathered}
f(\phi, t)=\int_{\Omega t}^{\Omega t+\pi} I(\lambda, \phi) L\left(\mu, \mu_{0}\right) \cos \lambda d \lambda, \\
F(t)=\int_{-\frac{\pi}{2}}^{\frac{\pi}{2}} f(\phi, t) \cos ^{2} \phi d \phi \\
L\left(\mu, \mu_{0}\right)=I_{0} \cdot\left(\mu \mu_{0}\right)^{k} / \mu
\end{gathered}
$$

where $f(\phi, t)$ is the rotational modulation of the flux at each latitude at time $t, \Omega$ is the rotation rate of Jupiter, $\lambda$ is the longitude, $\phi$ is the latitude, and $F(t)$ is the disk-integrated flux. In the reflection limb-darkening formula, $L\left(\mu, \mu_{0}\right), \mu$ is the cosine of the emission angle; $\mu_{0}$ is the cosine of the sunlight incident angle, which is fixed to be 1 (incident angle is $0^{\circ}$ ) in our light-curve construction; and $k$ is the limb-darkening coefficient for each map from the OPAL data set (Table 1).

\subsection{Thermal-emission Light-curve Construction}

In order to construct the thermal-emission light curves, we construct the cylindrical global maps without limb darkening in the following steps. First, we use the raw data reduction 
method presented in Fletcher et al. (2009) to reduce the data and assign latitude, longitude, and emission angle to each pixel. Second, we fit limb darkening in the mosaics at different wavelengths using the limb-darkening formula,

$$
L(\mu)=\left(c_{0}+c_{1} \mu\right) /\left(c_{0}+c_{1}\right),
$$

where $\mu$ is the cosine of the emission angle, $c_{0}$ is the zero-order coefficient, and $c_{1}$ is the first-order coefficient. We use the maximum-likelihood estimation (MLE) method to fit the limb darkening. Because the radiances of the pixels with larger emission angles (i.e., smaller $\mu$ ) usually have larger observational uncertainties, only the pixels with $\mu$ larger than 0.4 are selected in the limb-darkening fitting. We split $\mu$ from 0.4 to 1 into six uniform intervals with an interval width of 0.1 and randomly pick 50 pixels from each interval from $-70^{\circ} \mathrm{S}$ to $+70^{\circ} \mathrm{N}$. Then, we fit the limb-darkening formula using a Monte Carlo Markov chain package, EMCEE (Foreman-Mackey et al. 2013). The free parameters are limb-darkening coefficients, $c_{0}$ and $c_{1}$. The MLE equation is given by

$$
\begin{gathered}
\ln p\left(L_{0} \mid \mu, c_{0}, c_{1}, f_{1}, f_{2}\right) \\
=-\frac{1}{2} \sum^{n}\left[\frac{\left(L_{0}\left(\mu_{n}\right)-c_{1} \mu_{n}-c_{0}\right)^{2}}{s_{n}^{2}}+\ln \left(2 \pi s_{n}^{2}\right)\right], \\
s_{n}^{2}=f_{1}^{2} L_{0}\left(\mu_{n}\right)^{2}+f_{2}^{2}\left(c_{1} \mu_{n}+c_{0}\right)^{2},
\end{gathered}
$$

where $L_{0}\left(\mu_{n}\right)$ is the pixel radiance, and $\mu_{n}$ is the cosine of the emission angle of a selected pixel. According to the differences between the calibration of IRIS and CIRS on Jupiter (calibration uncertainty is about 5\%-10\%), the systematic uncertainties of the radiance, $f_{1}$ and $f_{2}$, are both chosen to be $10 \%$ in this study. We allow the term $f_{2}^{2}\left(c_{1} \mu_{n}+c_{0}\right)^{2}$ to change in the MCMC fitting. An example of limb-darkening fitting at $8.80 \mu \mathrm{m}$ is shown in Figure 2. Adopting a larger pixel uncertainty value (e.g., 20\%-50\%) does not change the light curves; only the uncertainty of the resulting light curves (shaded region in the lower panel of Figure 2) becomes larger. But it does not alter our conclusions in this study. We also tested fitting the limb darkening of belts and zones separately, but the constructed light curves do not change much either.

For each wavelength, we combine the individual cylindrical maps obtained from disk images (Figure 1) into a global radiance map, and then we remove the limb-darkening effect from each pixel. We have to deal with the overlapping area where two or more individual maps overlap. The traditional way is to first remove the limb darkening for each individual map and then average the data in the overlapping area (e.g., Fisher et al. 2016). But we note that pixel radiances with smaller $\mu$ usually have larger observational uncertainties, as well as larger cylindrical projection errors. To reduce the errors, in this study, we first combine the individual maps without removing the limb darkening. In the overlapping area, we select the pixels with maximum radiances. ${ }^{7}$ Then, we remove the limb-darkening effects of the final combined cylindrical maps to obtain a global radiance map in Figure 4.

Lastly, we construct the thermal-emission light curves from the global radiance maps using Equations (1), (2), and (4) to

\footnotetext{
We also tried another metric and selected the pixels with maximum $\mu$, but the two do not make a big difference because the pixel with a larger $\mu$ usually has a larger radiance.
}

(a)

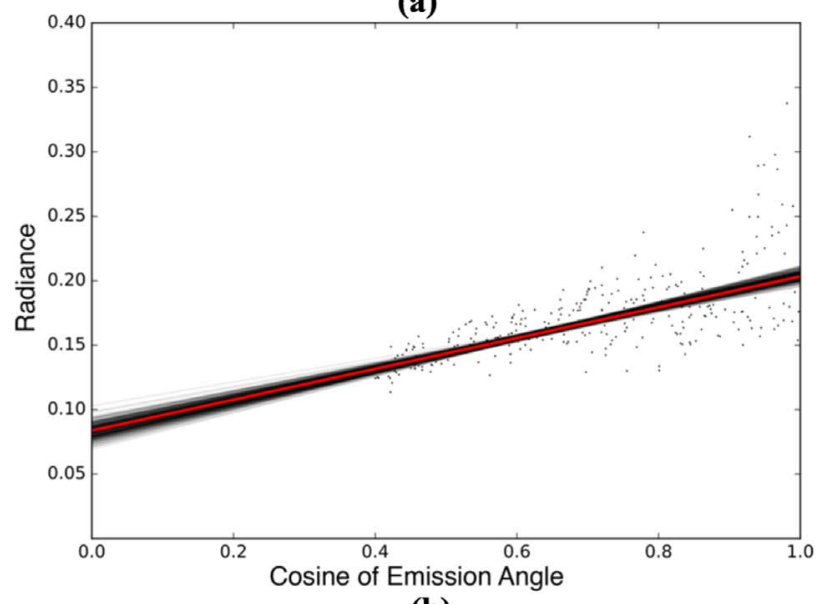

(b)
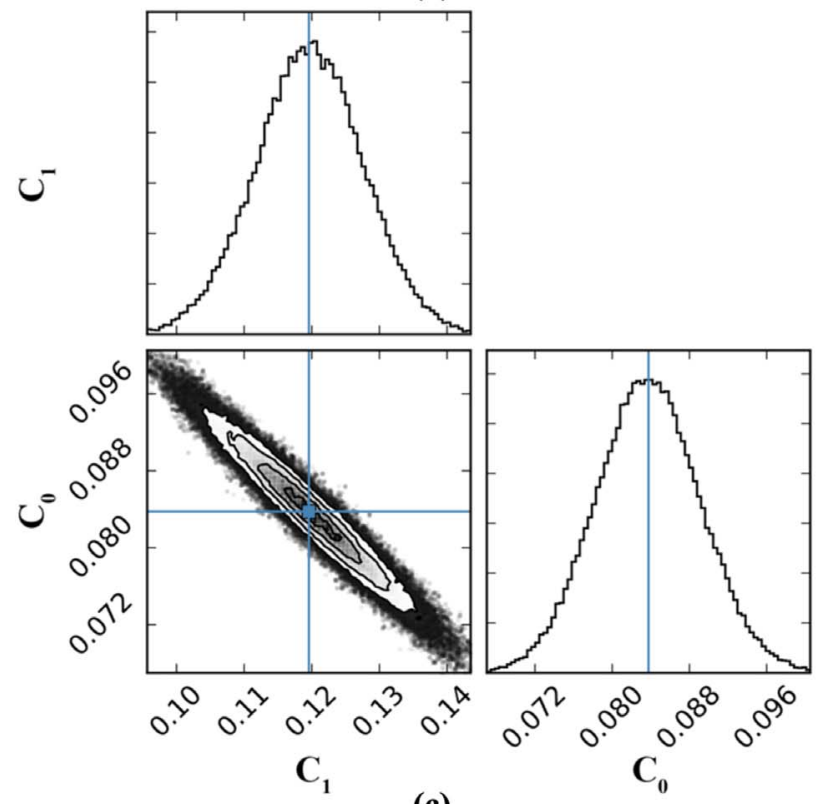

(c)

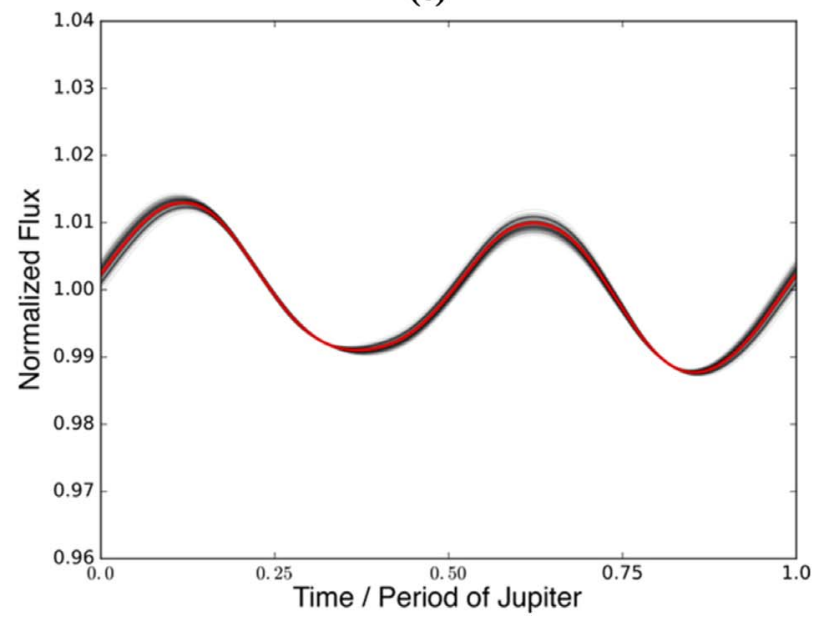

Figure 2. Example of limb-darkening fitting at $8.80 \mu \mathrm{m}$. (a) Limb-darkening profile. The dots are randomly selected 300 pixels from all latitudes with a cosine of the emission angles larger than 0.4. The red line denotes the fitting with the 50th percentile of the samples in the marginal distribution. (b) Posteriors of the fitting coefficients. (c) Uncertainty of the light-curve shape at $8.80 \mu \mathrm{m}$. The red curve is constructed by the red fitted line in panel (a). Black curves are constructed by the black fitted lines in panel (a). 


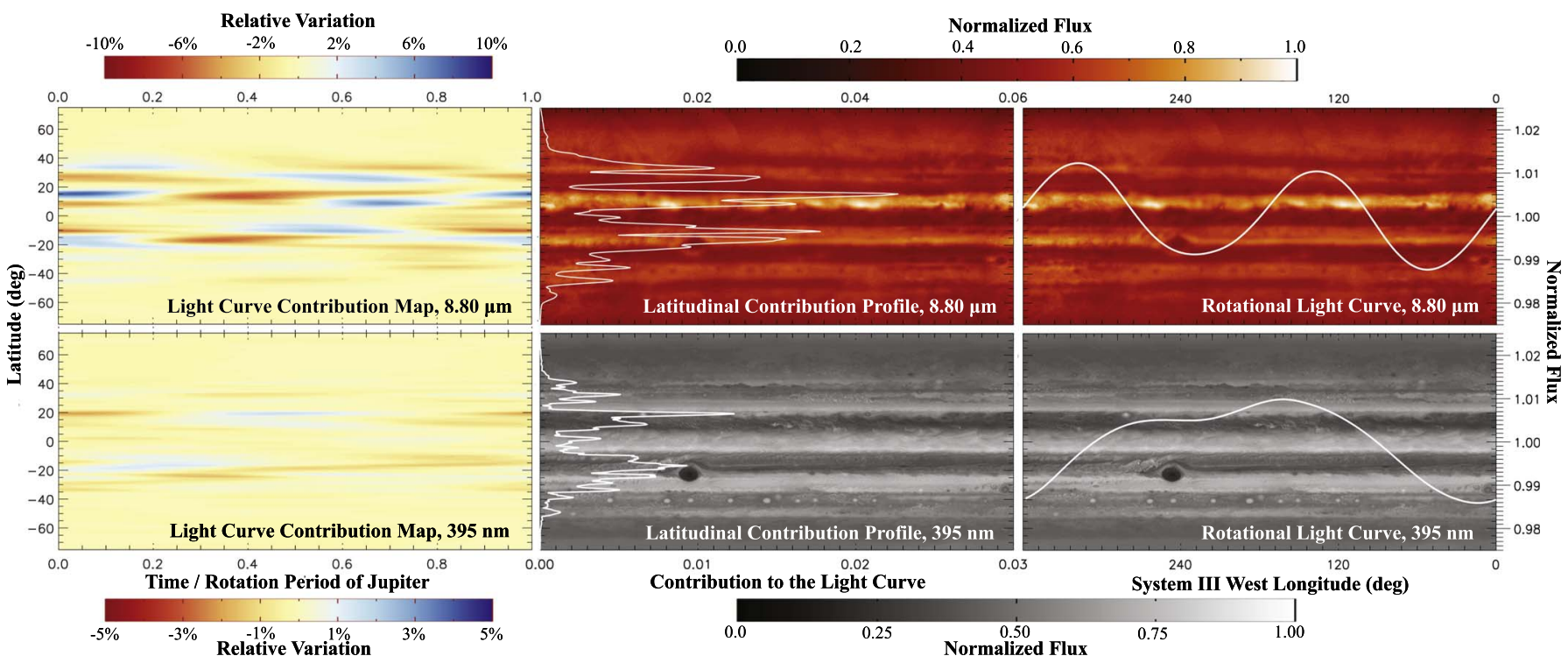

Figure 3. Light-curve contribution maps (left column), maps with latitudinal contribution profiles (middle column), and global radiance map with rotational light curves (right column) at $395 \mathrm{~nm}$ (lower row) and $8.80 \mu \mathrm{m}$ (upper row). The light-curve contribution maps and latitudinal contribution profiles indicate that the rotational variation is primarily exhibited in the belts and interfaces between the neighboring belts and zones. The light-curve contribution profiles are also correlated with the global radiance map. The variations (e.g., red and blue regions), which are induced by the GRS and the NEB expansion events, can be identified on the lightcurve contribution maps.

reintroduce the thermal-emission limb darkening $L(\mu)$ at every longitude. The limb-darkening removal and reintroduction could introduce uncertainties to the light curves. We quantify the uncertainty of the light-curve shape in Figure 2(c). The uncertainty of limb-darkening fitting influences the light-curve shape, but the effect is generally small.

\subsection{Contribution from Each Latitude}

We also develop a method to identify the locations of discrete patterns that primarily cause the rotational variability. First, instead of calculating the light curve for the entire globe, we construct the light curve at each latitude using Equation (1). Then, we define the contribution of latitude $\phi$ at time $t$ to the global light curve as

$$
C(\phi, t)=f(\phi, t) \cos ^{2} \phi / \sigma[F(t)],
$$

where $\sigma[F(t)]$ is the standard deviation of the global light curve, which represents the mean amplitude of the photometric variability.

We then construct the light-curve contribution maps and latitudinal contribution profiles from the latitude $-75^{\circ} \mathrm{S}$ to $+75^{\circ} \mathrm{N}$ (left and middle panels of Figure 3). Note that the positive and negative contributions from different latitudes could cancel each other out. Then, we calculate the standard deviation of $C(\phi, t)$ of each latitude, which can be considered as the mean contribution of latitude $\phi$ to the amplitudes of the global light curve. The latitudinal contribution profiles are shown in the middle panels of Figure 3 and left panels of Figure 4.

The major discrete patterns induce large rotational variabilities in the belts in the light-curve contribution map (Figure 3). For example, one can see the rotational variations caused by the GRS, NEB expansion event, and hot spots (i.e., blue regions caused by the bright patterns, red regions caused by the dark patterns) in the light-curve contribution map (Figure 3). Note that this method is only applicable to the equator-on-observed atmospheres because we do not include any information on inclination angles here. Next, we will describe these discrete patterns in detail.

\section{Discrete Patterns on Jupiter's Reflection and Thermal- emission Maps}

Jupiter's brightness maps from the UV $(275 \mathrm{~nm})$ to the midIR $(10.50 \mu \mathrm{m})$ are presented in Figure 4. The left panels show the cylindrical global maps with the latitudinal contribution profiles. The right panels are the same global radiance maps displayed with the corresponding light curves. Jupiter's global maps appear to vary significantly at different wavelengths because observations at different wavelengths probe into different pressure levels, and they are sensitive to different weather patterns. For example, Jupiter's banded structure is not evident on the UV $(275 \mathrm{~nm})$ and methane-band $(889 \mathrm{~nm})$ maps, but belts and zones are distinct at visible and thermal-emission wavelengths. For another example, the GRS is a dark spot in the visible-wavelength maps but a bright spot in the methaneband $(889 \mathrm{~nm})$ maps. These differences are controlled by distributions of temperature, chemical composition, hazes, and clouds in the troposphere and stratosphere.

Jupiter's atmosphere is generally dominated by the banded structure known as belts and zones. Belts are dark regions in reflected sunlight but appear bright and warm in thermal emission (Figure 5). They are cloudless, hot, and dry regions exhibiting several periodic events, such as the North Equatorial Belt (NEB) expansion events and fades and revivals of the South Equatorial Belt (SEB; e.g., Ingersoll et al. 2004; Fletcher et al. 2017a, 2017b). Zones are bright regions in reflected sunlight but appear dark in thermal emission (Figure 5). They are cloudy, cold, moist, and nearly uniform in longitude. Belts and zones play different roles in the rotational modulations of Jupiter. The latitudinal contribution profiles (left panels of Figure 4) indicate that belts exhibit much larger rotational modulations than zones at all wavelengths.

There are many discrete patterns in Jupiter's atmosphere (Figures 4 and 5). Here we discuss the most important ones. 


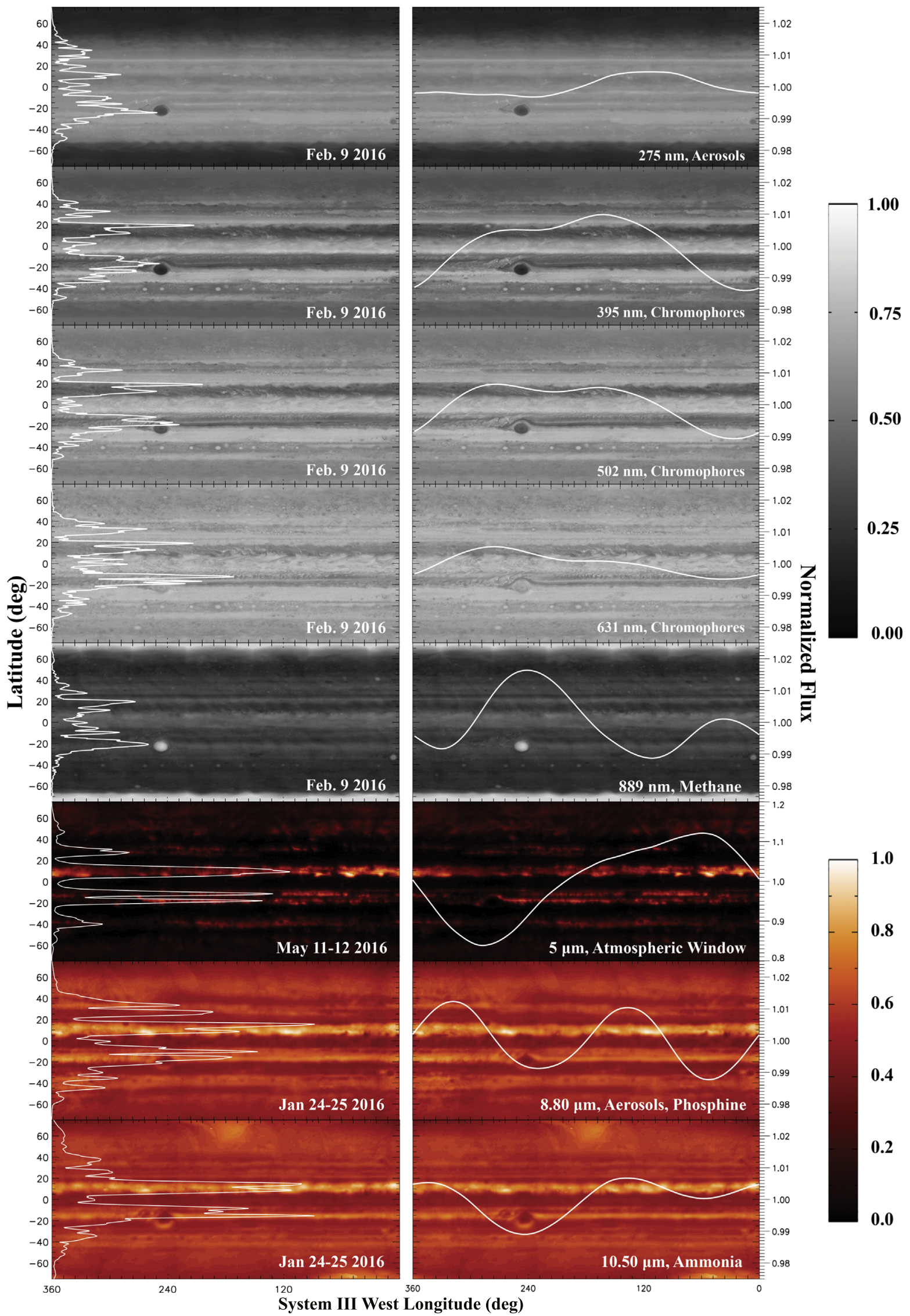

Figure 4. Jupiter's global radiance maps from the UV to mid-IR wavelengths, latitudinal contribution profiles (white lines in the left column), and rotational light curves (white lines in the right column). The latitudinal contribution profiles indicate that the belts exhibit more rotational modulations than the zones. The reflection latitudinal contribution profiles range from 0 to 0.03 ; the thermal-emission profiles range from 0 to 0.06 . 


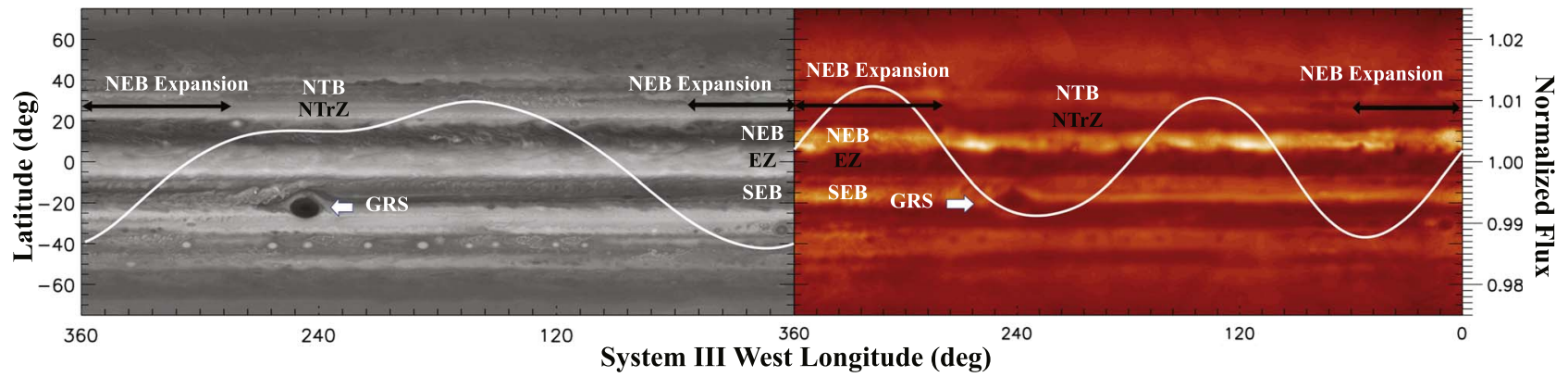

Figure 5. Locations of dominant patterns, periodic events, belts, and zones on typical Jupiter maps. The left panel is a reflected sunlight map imaged by WFC3/HST. The right panel is a thermal-emission map at $8.8 \mu \mathrm{m}$ imaged by COMICS/Subaru. The rotational light curve is also shown in each plot to illustrate the correlation between discrete patterns and the light-curve shape. Note that the peaks and troughs of the light curves do not precisely coincide with the location of the GRS and the NEB expansion event.

(1) The GRS. The GRS is a stationary vortex embedded in the boundary between the SEB and the South Tropical Zone (STrZ). It was located at latitude $22^{\circ} \mathrm{S}$ and longitude about $245^{\circ} \mathrm{W}$ in early 2016 (Figure 1), but the location of the GRS is not fixed on Jupiter. It longitudinally drifts along the southern interface of the SEB with a speed of $\sim 2 \mathrm{~m} \mathrm{~s}^{-1}$, which is about $\sim 120^{\circ} \mathrm{yr}^{-1}$ (Simon-Miller et al. 2002; Simon et al. 2018). The GRS is a region with enriched $\mathrm{NH}_{3}$ clouds and a high cloud top. Rising motions within the GRS are thought to bring gases $\left(\mathrm{NH}_{3}\right.$, $\mathrm{PH}_{3}, \mathrm{H}_{2}$ with a low para-fraction; e.g., Fletcher et al. 2010) up from the deeper troposphere, and adiabatic expansion causes the GRS to be cold at the cloud tops. It was proposed that at the cloud top, $\mathrm{NH}_{3}$ photochemically reacts with the hydrocarbons (e.g., $\mathrm{CH}_{4}, \mathrm{C}_{2} \mathrm{H}_{2}$ ) to produce chromophores that absorb UV and shortwavelength visible radiation (e.g., Ferris \& Ishikawa 1987; Carlson et al. 2016). Thus, on visiblewavelength maps, the GRS appears as a darker spot at shorter wavelengths (e.g., blue-absorbing chromophores) and brighter at longer wavelengths (high-altitude hazes; see reflection maps in Figures 4 and 5). The additional gaseous and aerosol absorbers from the upwelling, combined with the cold upper tropospheric temperatures, make the GRS appear dark and cold at thermal IR wavelengths.

(2) The NEB expansion event. The NEB spans $7^{\circ} \mathrm{N}-17^{\circ} \mathrm{N}$ planetographic latitude and appears dark in reflected sunlight and bright (i.e., warm and cloud-free) in thermalemission maps. The NEB periodically expands and contracts with a 3-5 yr life span, and our early 2016 observations captured once such event (Fletcher et al. 2017b). As the expansion event occurs, the NEB expands northward into the North Tropical Zone (NTrZ), but the physical mechanism behind the NEB expansion event is not well understood; it may result from subsidence and cloud clearing in the NTrZ associated with wave patterns on the northern edge of the NEB.

(3) Patchy cloud patterns in the North Temperate Belt (NTB). Patchy clouds in the NTB $\left(24^{\circ} \mathrm{N}-31^{\circ} \mathrm{N}\right)$ can be seen on the maps from 2015 to 2017 (Figures 4 and 5). In 2016 February, the dark-colored materials were distributed along the longitude ranging from $\sim 170^{\circ} \mathrm{W}$ to $\sim 280^{\circ}$ $\mathrm{W}$ (reflection maps in Figures 4 and 5). The white cloud patterns and brown chromophores interweave with each other in the NTB (visible-wavelength maps in Figures 4 and 5). The physical mechanism behind the intermittent
NTB cloud is not well understood but is likely related to the regular plume outbreaks on the southern edge of the NTB (Sánchez-Lavega et al. 2008, 2017), the most recent of which occurred in 2016 October.

(4) The SEB outbreak. Although no major fade-and-revival cycles of the SEB have been detected since 2009-2011, smaller outbreaks of plumes do sometimes occur in the mid-SEB. One such outbreak occurred in 2017 April at the longitude of $\sim 250^{\circ} \mathrm{W}$ (Figures 4 and 5). The SEB outbreak was bright in the visible wavelengths, caused by the strong reflection of the enhanced local fresh clouds, but appears dark in the thermal IR due to excess aerosol opacity and low temperatures associated with adiabatic expansion and cooling at the plume tops (Fletcher et al. 2017a).

(5) Thermal waves and hot spots in the NEB. Jupiter's midNEB exhibits longitudinal thermal wave patterns with wavenumbers of 10-17 (Orton et al. 1991; Deming et al. 1997; Li et al. 2006; Fletcher et al. 2017b), where an undulating pattern of warm and cool spots is anticorrelated with dark and bright patches of reflected sunlight. This has been interpreted as aerosols condensing in the cooler upwelling regions of a Rossby wave and sublimating in the warmer subsiding branches. This wave pattern can be seen to be modulating the mid-NEB thermal emission at $10.3-10.5 \mu \mathrm{m}$ in Figure 1. In addition, the $5 \mu \mathrm{m}$ hot spots are bright regions in thermal-emission wavelengths at latitudes $7^{\circ} \mathrm{N}-10^{\circ} \mathrm{N}$, the interface between the NEB and the Equatorial Zone (EZ). The $5 \mu \mathrm{m}$ hot spots are a chain of cloud-free, cyclonic features controlled by a westward-propagating Rossby wave on the boundary between the EZ and the NEB (Showman \& Dowling 2000). Although the $5 \mu \mathrm{m}$ hot spots are mainly controlled by large-wavenumber Rossby waves, they are not equally bright; some hot spots are significantly brighter than others. The brightness temperatures of warm regions (i.e., $5 \mu \mathrm{m}$ hot spots) are about $240-260 \mathrm{~K}$, meaning that the $5 \mu \mathrm{m}$ observation probes the effective pressure level at $\sim 3$ bars (Section 5.2.3 in West et al. 2004 and Figure 3 in Fletcher et al. 2016). Hot spots undergo different dynamical processes from those in the NEB. Numerical simulations suggest that there are strong vertical downdrafts in the hot spots, which could extend to at least a few bars. The downdrafts deplete the volatiles in this region (Showman \& Dowling 2000). Thus, $5 \mu \mathrm{m}$ hot spots are considerably brighter than the other regions on $5 \mu \mathrm{m}$ maps (Figures 4 
and 5). Interestingly, Figure 4 in Fletcher et al. (2016) shows the overlaps between several $5 \mu \mathrm{m}$ hot spots and warm airmasses at 8.80 and $10.50 \mu \mathrm{m}$. It is possible that the wave patterns (i.e., hot spots, warm airmasses) are controlled by the same group of Rossby waves.

(6) Small-scale patterns in belts and zones. There are numerous small-scale discrete patterns, such as anticyclonic white ovals near latitude $40^{\circ} \mathrm{S}$ and small cyclonic and anticyclonic patterns in the NEB and SEB. They ubiquitously exist in Jupiter's belts and zones. Although each individual small-scale pattern is too small to generate a large rotational variability, their total contribution to the rotational modulation cannot simply be neglected. According to the latitudinal contribution profiles (left panels in Figures 4 and 5), we crudely estimate that the contribution from the small-scale patterns is about $20 \%-30 \%$. However, because it is difficult to identify each individual pattern on the maps, we did not include them in the light-curve analysis in this study. Instead, the rotational modulations generated by these small-scale patterns are treated as the background variations.

Other than the horizontal structures discussed above, the vertical structure of Jupiter's atmosphere is another essential parameter to understand the rotational light curves at multiple wavelengths, because observations at different wavelengths could probe different altitudes. As we shall see later, the vertical structures of temperature, clouds (mainly $\mathrm{NH}_{3}$ clouds, possibly $\mathrm{H}_{2} \mathrm{O}$ and $\mathrm{NH}_{4} \mathrm{HS}$ clouds), hazes, and gases all play important roles in generating the rotational light curves of Jupiter. But their relative significances is wavelengthdependent.

\section{Jupiter's Light Curves}

Wavelength-dependent photometric variabilities are broadly exhibited by Jupiter's atmosphere from the UV to the mid-IR (Figure 4). The light curves also show various kinds of nonsinusoidal shapes at different wavelengths (Figures 4, 6, and 7). Figure 6 also shows a long-term light-curve evolution at reflection wavelengths over 3 yr. Figure 7 shows a short-term light-curve evolution at emission wavelengths over just a few weeks.

Based on Jupiter's maps, we can interpret the shapes, amplitudes, and evolution of Jupiter's light curves using the locations, sizes, brightness, and temporal evolution of discrete patterns in belts and belt-zone interfaces, such as the GRS and the NEB expansion regions. Our analysis quantitatively shows that light curves are controlled by the size, local temperature contrast, and cloud opacity with distributions of tropospheric gases, hazes, and chromophores. The light-curve evolution is likely to be caused by the temporal evolution of periodic events, such as the NEB expansion events, and drifting of the stationary vortices, such as the GRS and ovals.

\subsection{Light-curve Amplitude}

The light-curve amplitudes of Jupiter range from $~ 1 \%$ to $4 \%$ from the UV to the mid-IR wavelengths, except for the lightcurve amplitude at $5 \mu \mathrm{m}$, which exceeds $\sim 20 \%$, an order of magnitude larger than the others. This result is consistent with previous studies (Gelino \& Marley 2000; Karalidi et al. 2015). Based on the multiwavelength maps, latitudinal contribution

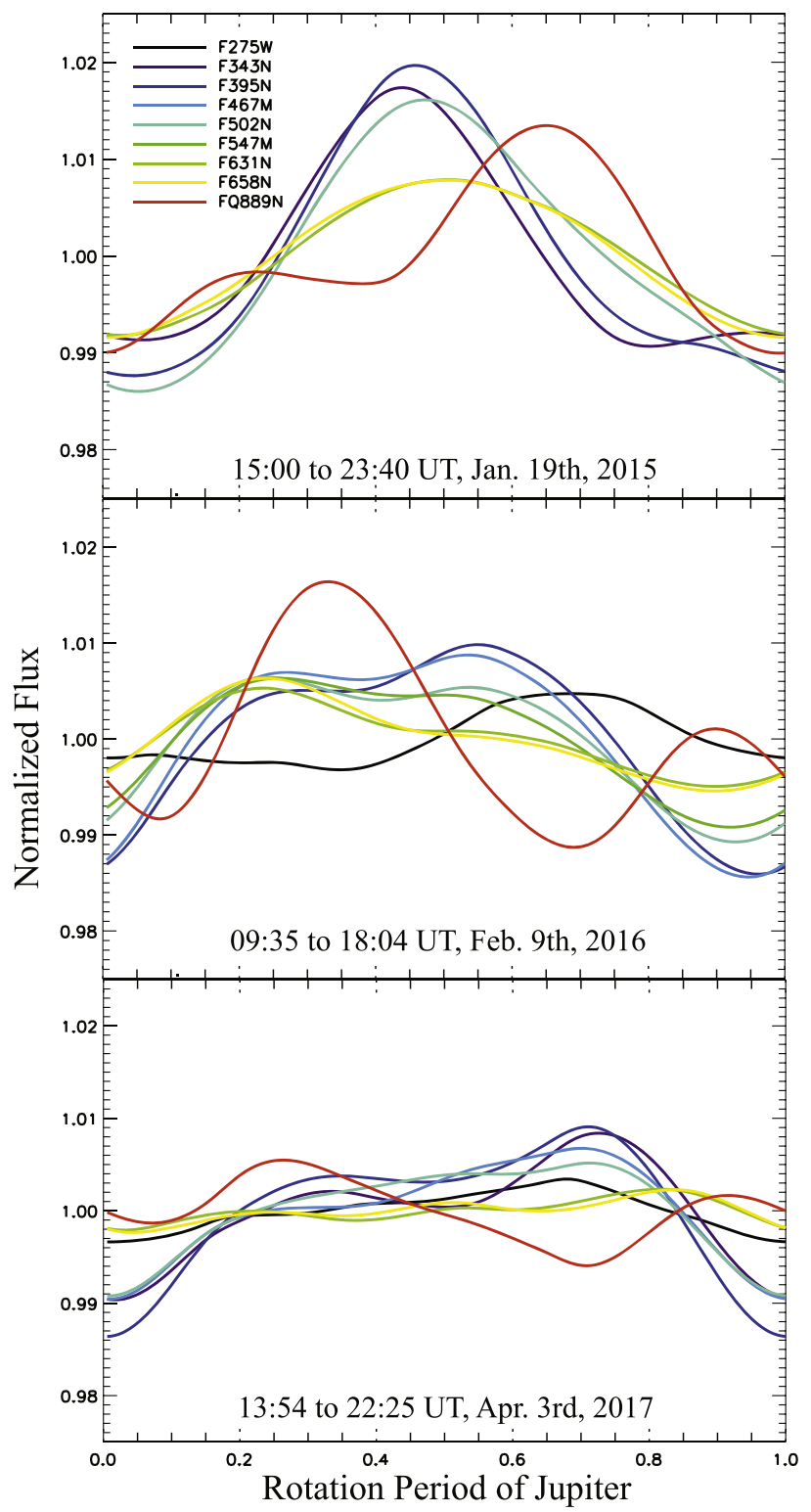

Figure 6. Light curves of Jupiter from 2015 to 2017 at reflection wavelengths. Most simultaneously observed light curves have similar shapes. The methaneband light curves (FQ889N) have obvious opposite-shape behavior compared with light curves at the other wavelengths. The amplitudes of the longwavelength (e.g., F658N) visible light curves are smaller than the shortwavelength ones (e.g., F343N).

profiles, and corresponding light curves, we can estimate the light-curve amplitudes by the sizes, brightness contrast ratios, and latitudes of the dominant patterns as

$$
A \sim S_{p} \cdot \Delta B \cdot \cos \theta,
$$

where $A$ is the light-curve amplitude; $S_{p}$ is the area fraction of the pattern over the disk; $\Delta B$ is the brightness contrast between the pattern and the background, which is given by $\left|B_{p} / B_{b}-1\right|$, where $B_{p}$ and $B_{b}$ are the brightness of the pattern and the background, respectively; and $\cos \theta$ is the projection factor of the pattern on the disk, where $\theta$ is the latitude of the discrete pattern.

The size of the individual discrete pattern is almost independent of wavelength. For example, the north-south width and east-west length of the GRS almost do not change at 


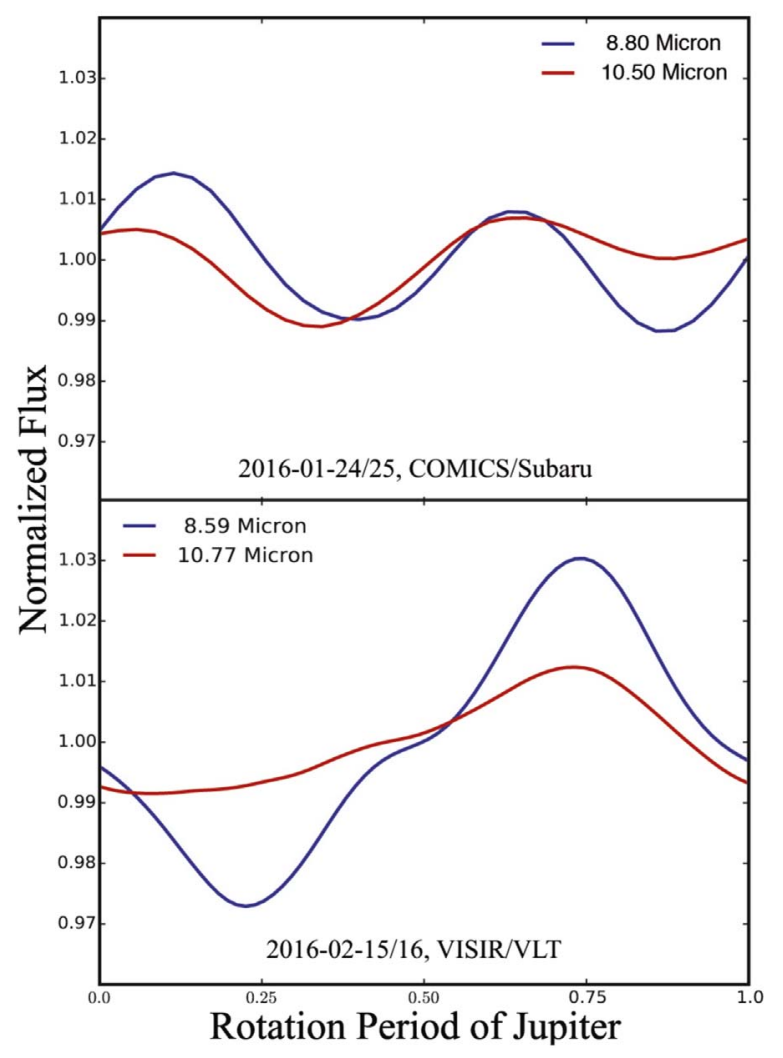

Figure 7. Jupiter's thermal-emission light curves separated by 3 weeks. The light-curve amplitude increases by a factor of 2 in a timescale of $~ 50$ Jupiter rotations. The locations of the peaks and troughs of the two light curves from the same observational date are similar. But their shapes evolve from a twopeak structure to a single-peak structure just after 3 weeks.

different wavelengths (Figure 4). However, the brightness contrast is highly wavelength-dependent. The mechanism behind the brightness contrast at thermal emission is different from that at reflection wavelengths. The reflection brightness contrasts between the discrete patterns and the background are controlled by the distributions of hazes, thin-thick clouds, and chromophores in and above the clouds. The brightness contrasts at thermal-emission wavelengths are mainly produced by the distributions of temperature, opacities of the tropospheric gases, hazes, and clouds.

The brightness contrasts of the patterns vary from $20 \%$ to $50 \%$ from the UV to the mid-IR, except for that at $5 \mu \mathrm{m}$, which can vary by a factor of $10(5 \mu \mathrm{m}$ hot spots; see color bars in Figure 5). The light-curve amplitude at $5 \mu \mathrm{m}$ is therefore significantly larger than any other wavelengths in this study. In the following sections, we first estimate the sizes of the discrete patterns. Then, we will focus on the mechanisms behind the wavelength-dependent brightness contrast.

\subsubsection{Size of the Weather Patterns}

The light-curve contribution maps and latitudinal contribution profiles (left panels in Figures 3 and 5) indicate that, in 2016 February, the important patterns were mainly located in the SEB, NTB, and northern and southern boundaries of the NEB. The corresponding discrete patterns are the GRS, patchy clouds in the NTB, the NEB expanded region (which does not cover all longitudes), and $5 \mu \mathrm{m}$ hot spots controlled by planetary waves, respectively. Here we focus on the most important two features: the NEB expansion event and the GRS.
When the NEB expansion occurred in 2016, the expanded region became one of the most important discrete patterns at both reflection and thermal-emission wavelengths (Figures 3 and 4). During the expansion event, the dark-colored materials in the NEB appeared to expand northward (see reflection maps in Figures 4 and 5) into the NTrZ (Fletcher et al. 2017b). Usually, the expansion occurs uniformly at all longitudes. But in 2016, only about one-third of the full circumference of the NEB was expanded northward $\left(\sim 50^{\circ} \mathrm{W}\right.$ to $\sim 290^{\circ} \mathrm{W}$ in the system III longitude; Fletcher et al. 2017b). This expansion region has a north-south width of $\sim 3^{\circ}$ and west-east length of $\sim 120^{\circ} \mathrm{W}$. The size, $S_{\mathrm{p}}$, of the NEB expansion region is about $3 \%$.

The GRS is important for the rotational modulation from the UV to the mid-IR on Jupiter. This is consistent with the results in Karalidi et al. (2015). The size of the GRS core is well measured. The north-south width and east-west length are about 17,000 and 39,000 km, respectively (Ingersoll et al. 2004). The size and brightness contrast of the whole GRS are not well constrained, because the GRS is not a simple dark spot on Jupiter maps (Figures 4 and 5). There are $\mathrm{NH}_{3}$ cloud spots and turbulent activities toward the northwest of the GRS, a region known as the "turbulent wake." This cloudy region is associated with the dynamic processes of the GRS (Palotai et al. 2014). Based on the bright GRS on the methane-band map (Figures 4 and 5), we estimate that the area fraction of the GRS, $S_{\mathrm{p}}$, is $5 \%$.

\subsubsection{Brightness Contrast at Reflection Wavelengths: Aerosols, Chromophores, and Methane}

The brightness contrasts of discrete patterns on reflection maps vary from $20 \%$ to $50 \%$ (see color bars in Figure 4). The reflection brightness is dominated by the reflection of $\mathrm{NH}_{3}$ clouds and the absorption of hazes, chromophores in the $\mathrm{NH}_{3}$ clouds, and the methane gas absorption above the clouds.

The haze absorption dominates the appearance and brightness contrast of Jupiter in the UV $(275 \mathrm{~nm})$. The GRS is darker than the background due to the particles on the cloud top of the GRS (West et al. 2004; Zhang et al. 2013; Carlson et al. 2016). At other latitudes, the distributions and brightness of upper tropospheric hazes are nearly uniform in longitude (UV map in Figure 4). Furthermore, the brightness contrast between belts and zones is less than $20 \%$, which is significantly smaller than that at other wavelengths (Figure 4). But the NEB expansion event could still be vaguely seen from longitude $\sim 80^{\circ} \mathrm{W}$ to $\sim 180^{\circ} \mathrm{W}$. Given that the dominant pattern covers about a few percent, the amplitude of the UV light curve is therefore less than $\sim 1 \%$ (Equation (8)).

The brightness contrast in the visible maps is dominated by chromophores in and above the $\mathrm{NH}_{3}$ clouds. Recent studies indicate that the reddish-brown chromophores ubiquitously exist in Jupiter's upper troposphere (Carlson et al. 2016; Sromovsky et al. 2017). The chromophores preferentially absorb short-wavelength visible light, and the absorbability generally decreases as the wavelength increases. The column density of chromophores in the NEB, SEB, and GRS is about $18-20 \mu \mathrm{g} \mathrm{cm}^{-2}$, while the column density in the EZ is about $13 \mu \mathrm{g} \mathrm{cm}^{-2}$ (Sromovsky et al. 2017). Because of abundant chromophores, belts and the GRS absorb more short-wavelength visible light than the zones. The brightness contrasts between belts and zones decrease from $\sim 50 \%$ at $395 \mathrm{~nm}$ to $\sim 20 \%$ at $658 \mathrm{~nm}$. Accordingly, because the pattern size is independent of 
wavelength, the light-curve amplitude decreases as wavelength increases (Figures 4 and 6).

The methane-band map is controlled by methane absorption, as well as $\mathrm{NH}_{3}$ cloud reflection. The effective pressure level at $889 \mathrm{~nm}$ is $\sim 0.6$ bar (West et al. 2004), corresponding to the $\mathrm{NH}_{3}$ cloud top in the EZ and the GRS. Aerosols above this level lead to more cloud reflection and less $\mathrm{CH}_{4}$ absorption. Thus, the EZ and GRS appear brighter than the NEB and SEB (see methane-band map in Figure 4). Because methane is well mixed, the spatial brightness contrast is mainly controlled by the spatial distribution of the $\mathrm{NH}_{3}$ clouds. The methane-band map shows that the brightness contrast between the GRS and the SEB is roughly $50 \%$, leading to a light-curve amplitude of $\sim 2 \%$. Furthermore, because belts and the GRS appear brighter than the surroundings at $889 \mathrm{~nm}$ instead of darker in the visible wavelengths, the shape of the $889 \mathrm{~nm}$ light curve is significantly different from the others. We will discuss the light-curve opposite-shape behaviors in methane-band light curves in Section 5.2.1.

\subsubsection{Brightness Contrast at Emission: Temperature versus Opacity}

At 8.80 and $10.50 \mu \mathrm{m}$, the radiance contrast is roughly $50 \%$ (Figures 3 and 4), implying that the brightness temperature contrast is about $6 \mathrm{~K}$ (Fletcher et al. 2016). However, at $5 \mu \mathrm{m}$, the radiance contrast could exceed a factor of 5 (Figure 4). The corresponding brightness temperature contrast is larger than $20 \mathrm{~K}$ (Fletcher et al. 2016).

The brightness temperature contrast is primarily controlled by the spatial variations of temperature, gases, and aerosols at 8.80 and $10.50 \mu \mathrm{m}$. The former wavelength is more sensitive to upper tropospheric hazes and phosphine, whereas the latter one is more sensitive to the tropospheric $\mathrm{NH}_{3}$ gas. As an example, here we quantitatively estimate the brightness temperature contrast induced by the horizontal distributions of $\mathrm{NH}_{3}$ gas and temperature at $10.50 \mu \mathrm{m}$. At the same pressure level, $\mathrm{NH}_{3}$ is not homogeneously distributed over the globe. The retrieved $\mathrm{NH}_{3}$ mixing ratio at $\sim 500$ mbar in the GRS and EZ is roughly 20-25 ppm, about twice that in the SEB and NEB (Fletcher et al. 2016). Therefore, the effective emission levels (where the gas optical depth is unity) in the $\mathrm{NH}_{3}$-enriched region (e.g., the GRS/NTrZ) is higher than that in the $\mathrm{NH}_{3}$-depleted region (e.g., the NEB/SEB). Observations show that the $\mathrm{NH}_{3}$ mixing ratio exponentially decreases from $100 \mathrm{ppm}$ at $700 \mathrm{mbar}$ to $0.1 \mathrm{ppm}$ at $250 \mathrm{mbar}$ (Showman \& de Pater 2005; Fletcher et al. 2016). Using this profile, we estimate that the height difference is about $2 \mathrm{~km}$ between the effective emission levels in the $\mathrm{NH}_{3}$-enriched and -depleted regions. Given the adiabatic lapse rate of $\sim 2 \mathrm{~K} \mathrm{~km}^{-1}$, the temperature difference between the two effective emission levels (due to the gas opacity contrast) is about 3-4 K. On the other hand, the horizontal temperature difference between the NEB and the NTrZ, as well as that between the GRS and the SEB, is also 3 K (Nixon et al. 2010; Fletcher et al. 2016) at this pressure level ( $~ 500$ mbar). Therefore, the total brightness temperature contrast between the GRS/SEB and the NEB/NTrZ induced by both gas opacity and horizontal temperature contrast is about 5-7 K. This leads to about $40 \%-60 \%$ brightness contrast at $10.50 \mu \mathrm{m}$ (see color bar in Figure 4). Given that the dominant pattern size, $S_{\mathrm{p}}$, is about $3 \%-5 \%$, the magnitude of the rotational modulations at $10.50 \mu \mathrm{m}$ is therefore $\sim 2 \%-4 \%$, in agreement with the observed rotational modulation at $10.50 \mu \mathrm{m}$ (Figures 4 and 6).
Observations at $5 \mu \mathrm{m}$ are considerably different from other thermal-emission wavelengths. The $5 \mu \mathrm{m}$ maps are mainly dominated by brightness contrast induced by vertical cloud structures. The latitudinal contribution profiles indicate that the $5 \mu \mathrm{m}$ hot spots provide a significant contribution to the lightcurve amplitude. Because at $5 \mu \mathrm{m}$, light can penetrate into the deep troposphere in the cloudless hot-spot region but cannot go through the thick $\mathrm{NH}_{3}$ cloud layers in zones, the observations roughly probe three different layers. The highest layer is the cloudy zones at 700 mbar with a thick $\mathrm{NH}_{3}$ cloud region. The middle layer is the belts with thin clouds deeper than that in the thick-cloud region (i.e., the GRS, zones). The deepest layer is the deep atmospheric layer seen in the $5 \mu \mathrm{m}$ hot spot at about 1-4 bars. Thus, the $5 \mu \mathrm{m}$ hot spots can be considered as $\mathrm{NH}_{3}$ cloud holes. The height difference between the effective pressure levels of $5 \mu \mathrm{m}$ hot spots and the background (i.e., interface between the NEB and the EZ) is larger than $\sim 10 \mathrm{~km}$. The corresponding temperature difference between two layers is larger than $20 \mathrm{~K}$, which is sufficient to produce a large brightness contrast and resultant large rotational modulation. Although there is no good observational constraint on the temperature distribution below the $\mathrm{NH}_{3}$ cloud layers, simulations suggest that the largest horizontal temperature contrast at the same pressure level is limited below $5 \mathrm{~K}$ (Lian \& Showman 2010). Therefore, we conclude that the large lightcurve amplitude at $5 \mu \mathrm{m}$ is generated from the $5 \mu \mathrm{m}$ hot spots (i.e., the $\mathrm{NH}_{3}$ cloud holes) and thin-thick cloud distributions instead of the horizontal temperature variation at the same pressure level.

\subsection{Light-curve Shape, Evolution, and Phase Shift}

\subsubsection{Light-curve Shape}

Most simultaneously imaged light curves have similar shapes but are often irregular instead of sinusoidal (Figures 6 and 7). At reflection wavelengths, the light curves in 2015 January showed one obvious peak near the longitude of $\sim 180^{\circ}$ W. But, in 2016 February and 2017 April, except for the methane band $(889 \mathrm{~nm})$, the light curves showed an obvious trough instead of a peak near the longitude of $\sim 30^{\circ} \mathrm{W}$. The methane-band light curve in 2016 February had two peaks near longitude $\sim 240^{\circ} \mathrm{W}$ and $\sim 30^{\circ} \mathrm{W}$ and two troughs at longitude $\sim 330^{\circ} \mathrm{W}$ and $\sim 110^{\circ} \mathrm{W}$. In 2017 April, it also had a two-peak structure.

The light-curve peaks and troughs are not precisely located at the same longitudes of the discrete patterns. For example, in 2016 February, at the visible wavelengths (Figure 8), the small light-curve trough at longitude $\sim 215^{\circ} \mathrm{W}$ did not coincide with the location of the GRS at $\sim 245^{\circ} \mathrm{W}$. In 2017 April (Figure 8), the trough of the visible light curve appeared at a longitude of about $\sim 0^{\circ} \mathrm{W}$, but the longitude of the GRS was at $\sim 25^{\circ} \mathrm{W}$. The reason is that none of the discrete patterns is simply dark or bright compared to the background (as noted in Section 5.1.1). For example, the bright clouds on the west limb of the GRS increase the total reflected flux and shift the light-curve trough eastward. The numerous small-scale structures in the belts and zones (Section 4, point 6), which are not well resolved in our current images, can also modulate light curves. In addition, the contributions from dark and bright patterns can cancel each other out. According to the light-curve contribution maps at $395 \mathrm{~nm}$ (Figure 3), the contribution from the GRS offsets that from the bright regions in the west limb of the GRS and the 


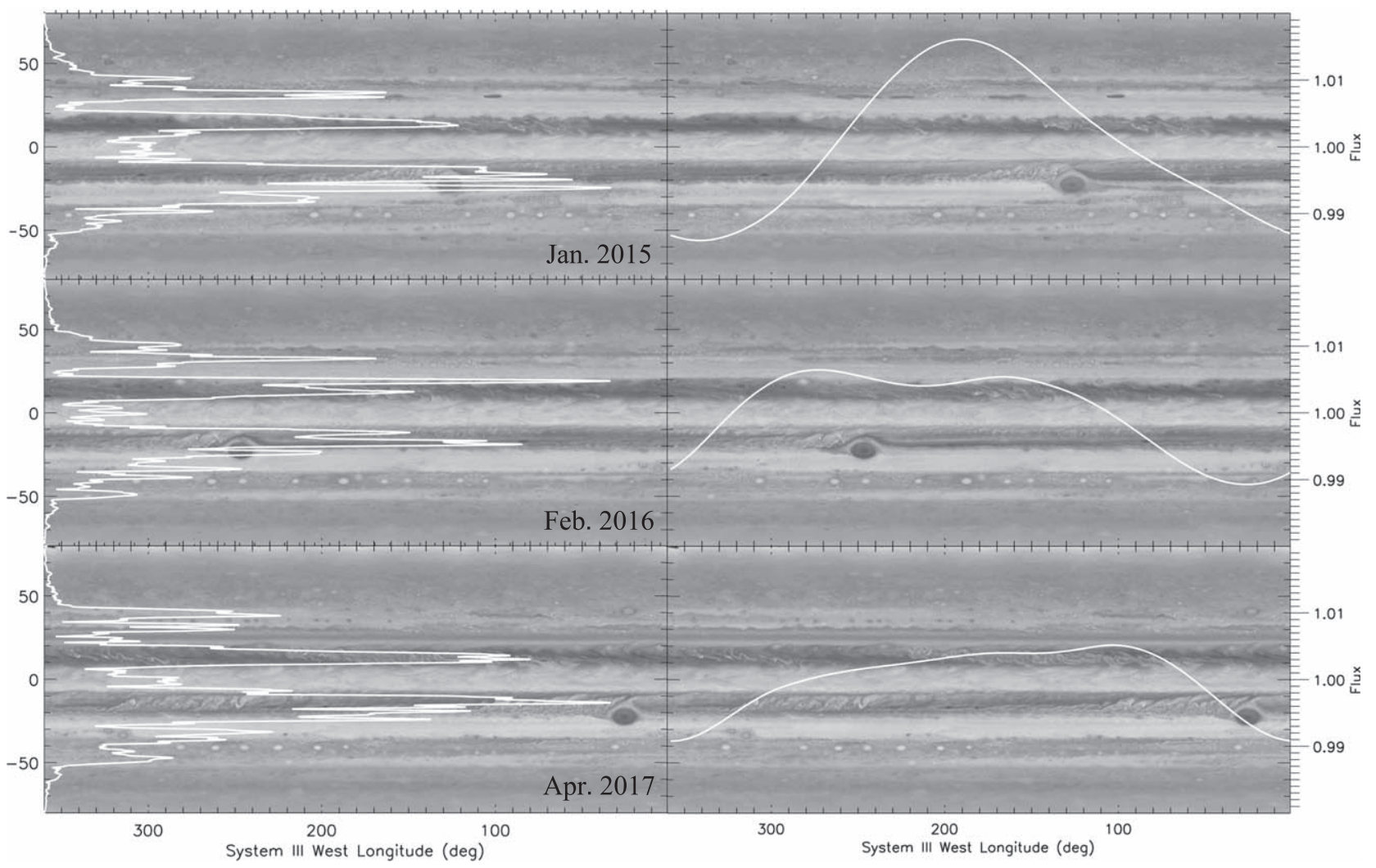

Figure 8. Long-term evolution of Jupiter's visible maps and rotational light curves (white lines in the right panels) at $395 \mathrm{~nm}$ from 2015 to 2017 . The latitudinal contribution profiles (white lines in the left panels) indicate that the contribution to the rotational modulation at each latitude does not change much with time. The maps also show the evolution of several periodic events (e.g., NEB expansion event, SEB outbreak) and the drifting of the GRS, as well as the drifting of the patchy clouds in the NTB. The occurrence and disappearance of the NEB expansion event, which do not progress equally over all longitudes, can also be seen from the latitudinal contribution profiles.

NEB expanded area (at the rotation period from 0.2 to 0.4 ). Thus, the locations of important discrete patterns cannot be precisely identified from the light-curve shapes.

\subsubsection{Light-curve Evolution}

Jupiter's light curves exhibit active short- and long-term evolution (Figures 6-8). The short-term evolution is seen during $\sim 50$ Jupiter rotations between 2016 January and February at thermal-emission wavelengths (Figure 7). The long-term evolution is seen at reflection wavelengths from 2015 to 2017 (Figures 6 and 8). The light-curve evolution is a result of the evolution of periodic events, such as the NEB expansion and SEB outbreak; the longitudinal drifting of the GRS; and the patchy clouds in the NTB. However, we do not have continuous long-term observations to unveil sufficient details about the short- and long-term light-curve evolution on Jupiter. More observations are needed in the future to quantify the underlying mechanisms.

\subsubsection{Wavelength Dependence}

As noted in Section 5.2.1, although most simultaneously imaged light curves exhibit similar shapes, the light curve at the methane-band wavelength $(889 \mathrm{~nm})$ is significantly different from that in the other wavelengths (Figures 4 and 6). For example, in 2015 January, 2016 February, and 2017 April, the methane-band light curves exhibit peaks where the light curves in the UV and visible wavelengths have troughs (Figure 6).
The opposite-shape behavior of the methane-band light curve is a result of the brightness change of the GRS at different wavelengths. Normally, the GRS appears dark at visible wavelengths due to chromophore absorption. However, because the methane absorption is very strong at $889 \mathrm{~nm}$, the GRS appears bright because there is less methane absorption above the high cloud top of the GRS. Therefore, when the GRS rotates into the view, the total flux decreases at visible wavelengths but increases at $889 \mathrm{~nm}$. As a result, the methane-band light curves exhibit an opposite behavior to that of the other wavelengths.

Light curves at different visible wavelengths also appear slightly differently (Figure 6). These behaviors are primarily induced by the chromophores. Because chromophores absorb more short-wavelength visible light (that is why they appear reddish), they create larger brightness contrasts between the chromophore-enriched patterns and background at shorter wavelengths.

The amplitudes of the two simultaneously observed light curves at thermal emission ( 8.80 and $10.50 \mu \mathrm{m}$; see Figure 7) are different by a factor of 2 , but the locations of peaks and troughs coincide with each other. A possible reason is that these two wavelengths probe roughly the same pressure level with similar distributions of temperature, aerosols, and gases $\left(\mathrm{NH}_{3}\right.$ and $\left.\mathrm{PH}_{3}\right)$. The shape of the $5 \mu \mathrm{m}$ light curve is also different from the other wavelengths. This might be expected because the underlying mechanism (i.e., cloud holes, thin-thick cloud structures) of the $5 \mu \mathrm{m}$ light curve is very different from the other wavelengths. But note that our $5 \mu \mathrm{m}$ images are taken 
at a different date, so the light-curve differences might also be a result of time evolution. More observations are needed in the future to investigate these behaviors in detail.

\section{Implications for Brown Dwarfs and Exoplanets}

It is believed that temperature perturbations and patchy cloud structures are responsible for the complex photometric rotational variabilities observed on brown dwarfs and directly imaged exoplanets (e.g., Marley et al. 2010; Buenzli et al. 2012; Apai et al. 2013; Morley et al. 2014; Esplin et al. 2016; Yang et al. 2016; Zhou et al. 2016; Apai et al. 2017). Weather patterns on brown dwarfs have been inferred by some indirect methods, such as mapping tools and Doppler imaging (Crossfield et al. 2014; Karalidi et al. 2015; Apai et al. 2017). However, without directly resolved images, it is difficult to understand the real mechanism underlying the rotational variability and light-curve evolution. This study of Jupiter's light curves could provide important insight on how the rotational modulations are produced in cloudy atmospheres. Here we list some implications on brown dwarfs and exoplanets from the perspectives of planetary waves, pattern size, the significance of temperature and opacity sources, and wavelength-dependent behavior.

\subsection{Patterns Controlled by Planetary-scale Waves}

Apai et al. (2017) suggested that the beating of planetary wavecontrolled patterns on brown dwarfs is a dominant cause for the rotational modulations and light-curve evolution. On Jupiter, the large-wavenumber (i.e., small-wavelength) patterns with unequally bright patterns, such as the bright bulges controlled by the tropospheric thermal waves (Ingersoll et al. 2004; Fletcher et al. $2017 \mathrm{~b}$ ) and $5 \mu \mathrm{m}$ hot spots controlled by Rossby waves, can generate rotational modulations at 5, 8.80, and $10.50 \mu \mathrm{m}$, respectively (Figure 4). At $5 \mu \mathrm{m}$, hot spots are the dominant patterns for the rotational variability. But note that highwavenumber planetary waves and associated weather patterns such as hot spots, if they have equal brightness, are not likely to induce a large rotational modulation because the brightness peaks and troughs tend to cancel each other out when averaged over the disk. Weather patterns with large-scale longitudinal asymmetry as seen around the NEB on Jupiter, which might be induced by lowwavenumber planetary waves or other complex meteorological activities, could also be responsible for the large rotational modulations on brown dwarfs and exoplanets.

\subsection{Pattern Size}

The GRS and the NEB expansion region (provided it does not progress to be longitudinally uniform) are found to be the important discrete patterns contributing to the rotational modulations of Jupiter. Our study shows that pattern size is very important in determining the magnitude of the photometric variability. Empirically, the pattern size on Jupiter is strongly related to the widths of belts and zones. As shown in Figures 4 and 5, the north-south width and the west-east length of the GRS are comparable to the width of the belts and zones. Because the NEB expansion event occurs between two adjacent belts and zones (the NEB and the NTrZ), the width of the expansion region should be smaller than the width of the bands. In an atmospheric mapping model, Aeolus, Karalidi et al. (2015) assumed that the diameter of the spot is equivalent to the width of the band. Zhang \& Showman (2014) suggested that the atmospheres of cold brown dwarfs could be dominated by the banded structure. If this were true, the bandwidth might be estimated by the Rhines scale $L_{\beta} \sim \pi(U / \beta)^{\frac{1}{2}}$, where $\beta$ is the Rossby parameter $2 \Omega \cos \phi / a, \phi$ is the latitude, $a$ is the planet radius, and $U$ is the characteristic flow speed (Rhines 1975; Vasavada \& Showman 2005). Therefore, the size of a large pattern might be empirically given by $L_{\beta} / a \sim \sqrt{\pi^{2} U / 2 \Omega a \cos \phi}$. For a Jupiter-size brown dwarf $\left(\sim 10^{5} \mathrm{~km}\right.$ ) with a typical spinning rate of several hr (Yang et al. 2016) and a flow speed of $\sim 100 \mathrm{~m} \mathrm{~s}^{-1}$ (see numerical simulation results in Showman \& Kaspi 2013; Zhang \& Showman 2014; Tan \& Showman 2017), we estimate that the size of a single discrete pattern might be smaller than $10 \%$ of the disk area. This is consistent with our Jupiter study: the GRS area fraction is about $5 \%$.

\subsection{Temperature versus Opacities of Gas and Clouds}

Previous studies only proposed patchy cloud opacity and temperature perturbations as a source for the rotational modulation on brown dwarf atmospheres (e.g., Apai et al. 2013; Morley et al. 2014; Zhang \& Showman 2014). On the other hand, our study of Jupiter shows that the distributions of gas, $\mathrm{NH}_{3}$ clouds, aerosols, and horizontal temperature in belts and zones are all important for the rotational modulations in thermal emission. The brightness contrast is primarily produced by the $\mathrm{NH}_{3}$ cloud holes and thin-thick clouds at $5 \mu \mathrm{m}$ (Section 5.1.3). The GRS, which is a localized high-cloudtop region, can generate rotational variability at all wavelengths. The GRS scenario is similar to the thin-thick cloud scenario proposed by Apai et al. (2013). But we found that the horizontal variations of gas opacity are also important for the thermal-emission light curves on Jupiter, a new mechanism that was not considered in previous brown dwarf studies.

On Jupiter, the distributions of temperature, gas opacity, and cloud opacity can significantly influence the light-curve amplitude. The rotational modulations at both 8.80 and $10.50 \mu \mathrm{m}$ are primarily controlled by the distributions of temperature and gas opacity. The light-curve amplitudes of Jupiter at two thermal-emission wavelengths are $2 \%-4 \%$. We note that the light-curve amplitudes of most variable brown dwarfs are also on the order of a few percent, implying that a similar mechanism - temperature and gas variation-might be applied to these low-amplitude-variability brown dwarfs. On the other hand, at $5 \mu \mathrm{m}$, the large light-curve amplitude ( $20 \%)$ of the rotational modulation is instead dominated by the cloud opacity variation (i.e., cloud holes, thin-thick clouds) because the $5 \mu \mathrm{m}$ observation is able to probe into the hot and deep atmosphere in the cloud-free region. Compared with the cases of 8.80 and $10.50 \mu \mathrm{m}$, the horizontal temperature variation is negligible to the rotational modulation at $5 \mu \mathrm{m}$. Some brown dwarfs (e.g., 2MASS2139, 2MASS1324) indeed show highamplitude light curves at certain wavelengths (Radigan et al. 2012; Heinze et al. 2015). We suggest that the underlying mechanism relevant to these brown dwarfs might be similar to that on Jupiter at $5 \mu \mathrm{m}$. We also note that the light-curve amplitudes of some brown dwarfs behave differently at different wavelengths (e.g., 2MASS2139 in Radigan et al. 2012; Apai et al. 2013). This might indicate that the relative significance of temperature and gas and cloud opacities is wavelengthdependent on these bodies, just like their behaviors on Jupiter.

Morley et al. (2014) discussed the contribution of temperature perturbation and patchy clouds to the rotational 
modulation. But note that the distributions of temperature, gas, and clouds could be highly correlated when the gas is condensable, because the condensable gas tends to form more clouds in cold regions, and clouds could have radiative feedback to modulate the local temperature. Alternatively, the gas abundances, clouds, and temperature distributions could all be controlled by vertical motions in convective atmospheres. For example, Jupiter's belts are less cloudy and warm, while zones are cloudy and cool. But even on Jupiter, why the clouds are enriched in zones and depleted in belts and how the cloud abundance is related to the banded structure are still not well understood.

\subsection{Light-curve Wavelength Dependence}

It is reported that some brown dwarfs exhibit wavelengthdependent rotational variabilities. The observations suggest that the "phases" of the light curves at some wavelengths are shifted compared with other wavelengths (e.g., Buenzli et al. 2012; Yang et al. 2016). On Jupiter, we do see interesting wavelengthdependent behaviors, such as various light-curve shapes and the peak shift of the methane-band light curve. However, we do not see an obvious "phase shift," because the multiwavelength lightcurve shapes are highly wavelength-dependent and significantly different from each other. The reason is that, as discussed in Karalidi et al. (2015), Jupiter's light curves have a much higher signal-to-noise ratio compared with brown dwarf observations, showing more detailed structures in their shapes. With more observations of brown dwarfs in the future, we may see more details of their light curves. We suggest that the mechanism behind the currently observed light-curve "phase shifts" on brown dwarfs is similar to what controls the wavelength-dependent behaviors (i.e., light-curve peak shift, opposite-shape behaviors) on Jupiter. For example, the observed "phase shifts" can be induced by the wavelength-dependent brightness of some patterns, such as the GRS on Jupiter.

\subsection{Inclination-angle Dependence}

The observed rotational modulation might also depend on the inclination angle to the observer. Here we define the inclination angle as zero for equator-on objects and $90^{\circ}$ for pole-on objects. We expect that on a low-inclined body, such as Jupiter, to an observer on the Earth, the spatial variations in the equatorial and tropical regions that contribute the most to the disk-integrated flux would be detectable because of rotational variations. For a planet observed pole-on, there is no variability in time that stems from rotational variations; only temporal modulations would be detected. Therefore, we would see the brightness modulations caused by different physical mechanisms. In fact, both the observations in Vos et al. (2017) and simulation results in Kostov \& Apai (2012) have shown that equator-on brown dwarfs have larger rotational modulations than pole-on.

\section{Conclusions and Discussion}

In this study, we constructed and analyzed Jupiter's rotational light curves from the UV to mid-IR wavelengths. The photometric variability and light-curve evolution are broadly exhibited at all wavelengths. Light-curve amplitudes and shapes are wavelengthdependent. In our analysis, the light-curve amplitudes vary from $1 \%$ to $4 \%$ at reflection wavelengths, $8.80 \mu \mathrm{m}$, and $10.50 \mu \mathrm{m}$, but the $5 \mu \mathrm{m}$ light-curve amplitude is more than $20 \%$. We used the latitudinal contribution profiles to identify the locations of the discrete patterns. The results show that the rotational modulations are primarily produced in the belts. Several important discrete patterns have been identified: (1) GRS, (2) the NEB expansion event in 2016 (which did not extend over all longitudes), (3) patchy cloud patterns in the NTB, (4) planetary wavecontrolled hot spots, (5) SEB outbreaks, and (6) small-scale patterns in belts and zones.

Our results show that the amplitudes of light curves are controlled by the brightness contrasts, sizes, and latitudes of the discrete patterns. The sizes of the dominant patterns (e.g., the GRS and the NEB expanded region) are about 3\%-5\% of Jupiter's disk area. The discrete pattern size is almost independent of wavelength.

We found that a large photometric variability tends to occur at a wavelength with a large brightness contrast on the map. At the reflection wavelengths, the spatial brightness contrast is controlled by tropospheric haze distribution, the absorption of chromophores, and patchy $\mathrm{NH}_{3}$ clouds. In the $\mathrm{UV}$, the brightness contrast is small due to the nearly uniform distributions of tropospheric hazes as a function of longitude. At the visible wavelengths, because chromophores absorb short-wavelength visible light and reflect long-wavelength light, the brightness contrast is smaller at longer wavelengths, leading to a smaller rotational modulation. In the methane band $(889 \mathrm{~nm})$, because the methane absorption is smaller in the high-cloud-top regions, the distributions of the patchy clouds, such as the GRS, dominate the brightness contrast.

In thermal emission, the brightness contrasts are generated by the inhomogeneous distributions of temperature, clouds, and gases. The relative significance of these factors is wavelengthdependent. At 8.80 and $10.50 \mu \mathrm{m}$, both the temperature contrast and gas opacity are important because the observations probe the pressure level above the main cloud layers. At the $5 \mu \mathrm{m}$ atmospheric window, observations can probe down to 1-4 bars in $\mathrm{NH}_{3}$ cloud holes and less than 1 bar in the thickcloud regions. The cloud opacity could therefore play a controlling role in the brightness contrast and the large rotational modulation at $5 \mu \mathrm{m}$.

We also found various light-curve shapes and short-term and long-term light-curve evolution on Jupiter. The shapes of the light curves are dominated by the locations and shapes of the patterns, such as the GRS, the NEB expanded region, and small-scale cyclonic patterns, in the NEB and SEB. But the light-curve peaks and troughs do not precisely coincide with the locations of the discrete patterns. The light-curve evolution is controlled by the temporally varying dynamic patterns, such as the drifting of the GRS, the periodic NEB expansion event, and the SEB outbreak event.

The light curves at the $889 \mathrm{~nm}$ methane band show an opposite-shape behavior compared with the light curves at the other wavelengths. The GRS appears as a bright spot in this methane band but as a dark spot at the UV and visible due to the high cloud top at the GRS. As a result, light curves in the methane band $(889 \mathrm{~nm})$ vary in the opposite sense to that at the other wavelengths.

Our study provides important insights into the study of the photometric variability on brown dwarfs and directly imaged exoplanets. We suggest the following. (1) If the brown dwarfs and planets are dominated by banded structures, and if we assume that the sizes of cloudy spots and band expansion are limited by the width of the bands, the size of the discrete patterns is probably smaller than the Rhines scale. (2) The distributions of the 
temperature, gas opacity, and patchy cloud opacity control the brightness contrast. For the wavelengths that sense the pressure levels above the main region of condensed volatiles, the distributions of temperature, gases, and aerosols should be important to the rotational modulation. This mechanism might be related to brown dwarfs with low-amplitude light curves. The cloud holes are more likely to produce large photometric variability at the wavelengths of atmospheric windows on brown dwarfs. We also pointed out that, because of the dynamical correlation (i.e., cooling or heating effects caused by the convections) between the cloud formation and temperature, there could be a degeneracy in the light-curve signal between the horizontal temperature contrast and patchy cloud opacity. (3) Bright and dark bulges with large-scale brightness structures could be the cause of the rotational modulations. These unequalbrightness sources might be induced by large-scale planetary waves and other weather activities. (4) For brown dwarfs observed pole-on, we should see less rotational variations and more dynamical variations; for brown dwarfs observed equatoron, we should see more rotational variations and a resultant larger light-curve amplitude.

Finally, in the future, multiwavelength (especially at the thermal wavelengths), continuous photometric monitoring of Jupiter for several successive rotations should provide more details on the underlying mechanisms of Jupiter's rotational light curves and their time evolution. It will further shed light on our interpretation of the rich observational data of rotational modulation on brown dwarfs and directly imaged exoplanets.

We thank Theodora Karalidi, Michael C. Liu and Ji Wang for helpful discussions. This research was supported by a NASA Earth and Space Science Fellowship to H.G., and NASA Solar System Workings grant NNX16AG08G and the Hellman Fellowship to X.Z. This research also benefited from the Outer Planetary Atmosphere Legacy project at https:// archive.stsci.edu/prepds/opal/. L.N.F. was supported by a Royal Society Research Fellowship and European Research Council Consolidator Grant (under the European Union's Horizon 2020 research and innovation program, grant agreement No. 723890) at the University of Leicester. G.S.O. and J.F. were supported by funds from NASA, distributed to the Jet Propulsion Laboratory, California Institute of Technology; J.F. was supported through JPL's Year-round Internship Program (YIP). This investigation was partially based on thermalinfrared observations acquired at (i) the ESO Very Large Telescope Paranal UT3/Melipal Observatory (program ID 096. C-0091); (ii) the Subaru Telescope and obtained from the SMOKA database, which is operated by the Astronomy Data Center, National Astronomical Observatory of Japan (program ID S16B-049); and (iii) NASA's Infrared Telescope Facility, which is operated by the University of Hawaii under contract NNH14CK55B with the National Aeronautics and Space Administration (program ID 2016A-022).

\section{ORCID iDs}

Huazhi Ge (葛華志) (1D https://orcid.org/0000-0001-6719-0759 Leigh N. Fletcher (iD https://orcid.org/0000-0001-5834-9588

\section{References}

Ackerman, A. S., \& Marley, M. S. 2001, ApJ, 556, 872

Apai, D., Karalidi, T., Marley, M., et al. 2017, Sci, 357, 683
Apai, D., Radigan, J., Buenzli, E., et al. 2013, ApJ, 768, 121

Bjoraker, G., Wong, M., de Pater, I., \& Ádámkovics, M. 2015, ApJ, 810, 122

Artigau, É., Bouchard, S., Doyon, R., \& Lafrenière, D. 2009, ApJ, 701, 1534 Buenzli, E., Apai, D., Morley, C. V., et al. 2012, ApJL, 760, L31

Burgasser, A. J., Marley, M. S., Ackerman, A. S., et al. 2002, ApJL, 571, L151 Carlson, R. W., Baines, K. H., Anderson, M., Filacchione, G., \& Simon, A. 2016, Icar, 274, 106

Cowan, N. B., \& Agol, E. 2008, ApJL, 678, L129

Crossfield, I., Biller, B., Schlieder, J., et al. 2014, Natur, 505, 654

Deming, D., Reuter, D., Jennings, D., et al. 1997, Icar, 126, 301

Esplin, T., Luhman, K., Cushing, M., et al. 2016, ApJ, 832, 58

Ferris, J. P., \& Ishikawa, Y. 1987, Natur, 326, 777

Fisher, B. M., Orton, G. S., Liu, J., et al. 2016, Icar, 280, 268

Fletcher, L., Orton, G., Yanamandra-Fisher, P., et al. 2009, Icar, 200, 154

Fletcher, L. N., Greathouse, T., Orton, G., et al. 2016, Icar, 278, 128

Fletcher, L. N., Orton, G., Mousis, O., et al. 2010, Icar, 208, 306

Fletcher, L. N., Orton, G., Rogers, J., et al. 2017a, Icar, 286, 94

Fletcher, L. N., Orton, G., Sinclair, J., et al. 2017b, GeoRL, 44, 7140

Foreman-Mackey, D., Hogg, D. W., Lang, D., \& Goodman, J. 2013, PASP, 125,306

Gelino, C., \& Marley, M. 2000, in ASP Conf. Ser. 212, From Giant Planets to Cool Stars, ed. C. A. Griffith \& M. S. Marley (San Francisco, CA: ASP), 322

Heinze, A. N., Metchev, S., Apai, D., et al. 2013, ApJ, 767, 173

Heinze, A. N., Metchev, S., \& Kellogg, K. 2015, ApJ, 801, 104

Ingersoll, A. P., Dowling, T. E., Gierasch, P. J., et al. 2004, in Jupiter: The Planet, Satellites and Magnetosphere, ed. F. Bagenal, T. E. Dowling, \& W. B. McKinnon (Cambridge: Cambridge Univ. Press), 105

Karalidi, T., Apai, D., Schneider, G., Hanson, J. R., \& Pasachoff, J. M. 2015, ApJ, 814, 65

Kataza, H., Okamoto, Y., Takubo, S., et al. 2000, Proc. SPIE, 4008, 1144

Kostov, V., \& Apai, D. 2012, ApJ, 762, 47

Lagage, P., Pel, J., Authier, M., et al. 2004, Msngr, 117, 12

Li, L., Ingersoll, A. P., Vasavada, A. R., et al. 2006, Icar, 185, 416

Lian, Y., \& Showman, A. P. 2010, Icar, 207, 373

Marley, M. S., Saumon, D., \& Goldblatt, C. 2010, ApJL, 723, L117

Metchev, S. A., Heinze, A., Apai, D., et al. 2015, ApJ, 799, 154

Morley, C. V., Fortney, J. J., Marley, M. S., et al. 2012, ApJ, 756, 172

Morley, C. V., Marley, M. S., Fortney, J. J., \& Lupu, R. 2014, ApJL, 789, L14

Morley, C. V., Skemer, A. J., Allers, K. N., et al. 2018, ApJ, 858, 97

Nixon, C. A., Achterberg, R. K., Romani, P. N., et al. 2010, P\&SS, 58, 1667

Orton, G. S., Friedson, A. J., Baines, K. H., et al. 1991, Sci, 252, 537

Palotai, C., Dowling, T. E., \& Fletcher, L. N. 2014, Icar, 232, 141

Powell, D., Zhang, X., Gao, P., \& Parmentier, V. 2018, ApJ, 860, 18

Radigan, J., Jayawardhana, R., Lafrenière, D., et al. 2012, ApJ, 750, 105

Rhines, P. B. 1975, JFM, 69, 417

Robinson, T. D., \& Marley, M. S. 2014, ApJ, 785, 158

Sánchez-Lavega, A., Orton, G., Hueso, R., et al. 2008, Natur, 451, 437

Sánchez-Lavega, A., Rogers, J. H., Orton, G., et al. 2017, GeoRL, 44, 4679

Showman, A. P., \& de Pater, I. 2005, Icar, 174, 192

Showman, A. P., \& Dowling, T. E. 2000, Sci, 289, 1737

Showman, A. P., \& Kaspi, Y. 2013, ApJ, 776, 85

Simon, A. A., Rowe, J. F., Gaulme, P., et al. 2016, ApJ, 817, 162

Simon, A. A., Tabataba-Vakili, F., Cosentino, R., et al. 2018, AJ, 155, 151

Simon, A. A., Wong, M. H., \& Orton, G. S. 2015, ApJ, 812, 55

Simon-Miller, A. A., Gierasch, P. J., Beebe, R. F., et al. 2002, Icar, 158, 249

Skemer, A. J., Morley, C. V., Allers, K. N., et al. 2016, ApJL, 826, L17

Sromovsky, L., Baines, K., Fry, P., \& Carlson, R. 2017, Icar, 291, 232

Stauffer, J., Marley, M. S., Gizis, J. E., et al. 2016, AJ, 152, 142

Tan, X., \& Showman, A. 2017, ApJ, 835, 186

Taylor, F., Atreya, S., Encrenaz, T., et al. 2004, in Jupiter: The Planet, Satellites and Magnetosphere, ed. F. Bagenal, T. E. Dowling, \& W. B. McKinnon (Cambridge: Cambridge Univ. Press), 59

Vasavada, A. R., \& Showman, A. P. 2005, RPPh, 68, 1935

Vincent, M. B., Clarke, J. T., Ballester, G. E., et al. 2000, Icar, 143, 189

Vos, J. M., Allers, K. N., \& Biller, B. A. 2017, ApJ, 842, 78

West, R. A., Baines, K. H., Friedson, A. J., et al. 2004, in Jupiter: The Planet, Satellites and Magnetosphere, ed. F. Bagenal, T. E. Dowling, \& W. B. McKinnon (Cambridge: Cambridge Univ. Press), 79

West, R. A., \& Smith, P. H. 1991, Icar, 90, 330

Yang, H., Apai, D., Marley, M. S., et al. 2016, ApJ, 826, 8

Zhang, X., \& Showman, A. P. 2014, ApJL, 788, L6

Zhang, X., West, R., Banfield, D., \& Yung, Y. 2013, Icar, 226, 159

Zhou, Y., Apai, D., Schneider, G. H., Marley, M. S., \& Showman, A. P. 2016, ApJ, 818, 176 